\title{
MEASURES ON COALLOCATION AND NORMAL LATTICES
}

\section{JACK-KANG CHAN}

Norden systems

75 Maxess Road

Melville, New York 11747, U.8.A.

( Received January 29, 1991 and in revised form April 15, 1991)

\section{ABSTRACT}

Let $\mathscr{L}_{1}$ and $\mathscr{L}_{2}$ be lattices of subsets of a nonempty set $X$. Suppose $\mathscr{L}_{2}$ coallocates $\mathscr{L}_{1}$ and $\mathscr{L}_{1}$ is a subset of $\mathscr{L}_{2}$. We show that any $\mathscr{L}_{1}$-regular finitely additive measure on the algebra generated by $\mathscr{L}_{1}$ can be uniquely extended to an $\mathscr{L}_{2}$-regular measure on the algebra generated by $\mathscr{L}_{2}$. The case when $\mathscr{L}_{1}$ is not necessary contained in $\mathscr{L}_{2}$, as well as the measure enlargement problem are considered. Furthermore, some discussions on normal lattices and separation of lattices are also given.

KEY WORDS : lattices, normal lattices, coallocation lattices, semi-separated lattices, regular finitely additive measures, $\sigma$-smooth measures, measure extension, measure enlargement.

1980 Mathematics Subject Classification: $28 \mathrm{C} 15,28 \mathrm{a} 12$.

\section{INTRODUCTION}

Let $X$ be an arbitrary set and $\mathscr{L}_{1}$ and $\mathscr{L}_{2}$ are lattices of subsets of $X$. If $\mathscr{L}_{1} \subset \mathscr{L}_{2}$, and if $\mathscr{L}_{2}$ coallocates $\mathscr{L}_{1}$, then any $\mathscr{L}_{1}$-regular finitely additive measure on the algebra generated by $\mathscr{L}_{1}$ can be uniquely extended to an $\mathscr{L}_{2}$-regular measure on the algebra generated by $\mathscr{L}_{2}$. This situation has been investigated by $J$. Camacho in [2]. We extend his results in several directions in this paper. We will consider the case where $\mathscr{L}_{1}$ is not necessary contained in $\mathscr{L}_{2}$ (see Theorem 3.1) and show that under suitable conditions any $\mu \in M_{R}\left(\mathscr{L}_{1}\right)$ (see below for definitions) gives rise to a $\nu \in M_{R}\left(\mathscr{L}_{2}\right)$. We will also 
consider besides measure extension problems, measure enlargement problems (see e.g. Theorem 3.3) and will finally apply these results to the case of a single lattice $\mathscr{L}$, thereby extending results of $M$. Szeto [8] for measures on normal lattices.

We begin by giving some standard lattice and measure theoretic background in section 2. Our notation and terminology is consistent with $[1,4,6,7,9]$. In section 3 , we consider the general coallocation theorem and a variety of consequences of it. Section 4 is devoted to a more detailed discussion of normal lattices and to separation of lattices. This work extends to some extent that of G. Eid [3].

\section{BACKGROUND AND TERMINOLOGY}

In this section, we summarize some lattice and measure theoretic notions and notations. This is all fairly standard and as previously mentioned is consistent with standard references.

\section{Definition 2.1}

Let $X$ be a nonempty set and $P(X)$ is the power set of $X$. A lattice $\mathscr{L}$ is a collection of subsets of $X$, which is closed under finite unions and finite intersections, and $\emptyset, X \in \mathscr{L}$. Let

$$
\mathscr{L}^{\prime} \equiv\left\{L^{\prime}: \quad L \in \mathscr{L}\right\}
$$

where $L^{\prime}$ denotes the complement of $\mathrm{L} . \mathscr{L}^{\prime}$ is a lattice if $\mathscr{L}$ is.

\section{Definition 2.2}

Let $\mathscr{L}, \mathscr{L}_{1}$ and $\mathscr{L}_{2}$ be any lattices of subsets of $\mathrm{X}$.

(1) $\mathscr{L}$ is $\delta$ if it is closed under countable intersections.

(2) $\mathscr{L}$ is a complement generated (c.g.) lattice if

$\forall \mathrm{L} \in \mathscr{L}, \exists \mathrm{L}_{1}, \mathrm{~L}_{2}, \ldots \in \mathscr{L}$ such that $\mathrm{L}=\bigcap_{\mathrm{n}=1}^{\infty} \mathrm{L}_{\mathrm{n}}^{\prime} \cdot$

(3) $\mathscr{L}$ is a normal lattice if

$\forall \mathrm{L}_{1}, \mathrm{~L}_{2} \in \mathscr{L}, \mathrm{L}_{1} \cap \mathrm{L}_{2}=\emptyset \Rightarrow$ $\exists \tilde{\mathrm{L}}_{1}, \tilde{\mathrm{L}}_{2} \in \mathscr{L}$ s.t. $\mathrm{L}_{1} \subset \tilde{\mathrm{L}}_{1}^{\prime}, \mathrm{L}_{2} \subset \tilde{\mathrm{L}}_{2}^{\prime}, \tilde{\mathrm{L}}_{1}^{\prime} \cap \tilde{\mathrm{L}}_{2}^{\prime}=\emptyset$.

(4) $\mathscr{L}$ is a countably paracompact (c.p.) lattice if

$\forall L_{1}, L_{2}, \ldots \in \mathscr{L}, L_{1} \supset L_{2} \supset \ldots, \lim _{n \rightarrow \infty} L_{n}=\emptyset\left(L_{n} \downarrow \emptyset\right) \Rightarrow$

$\exists \tilde{\mathrm{L}}_{1}, \tilde{\mathrm{L}}_{1}, \ldots \in \mathscr{L}$ s.t. $\forall \mathrm{n}, \mathrm{L}_{\mathrm{n}} \subset \tilde{\mathrm{L}}_{\mathrm{n}}^{\prime}$ and $\tilde{\mathrm{L}}_{\mathrm{n}} \downarrow \emptyset$.

(5) $\mathscr{L}_{2}$ is $\mathscr{L}_{1}$-countably-paracompact $\left(\mathscr{L}_{1}\right.$-c.p.) if 


$$
\begin{aligned}
& \forall B_{1}, B_{2}, \ldots \in \mathscr{L}_{2}, B_{1} \supset B_{2} \supset \ldots, B_{n} \downarrow \emptyset \Rightarrow \\
& \exists A_{1}, A_{2}, \ldots \in \mathscr{L}_{1} \text { s.t. } \forall n, B_{n} \subset A_{n}^{\prime} \text { and } A_{n}^{\prime} \downarrow \emptyset .
\end{aligned}
$$

(6) $\mathscr{L}_{1}$ semi-separates $\mathscr{L}_{2}$ if

$\forall A \in \mathscr{L}_{1}, \quad B \in \mathscr{L}_{2}, \quad A \cap B=\emptyset \Rightarrow \exists \tilde{L}_{1} \in \mathscr{L}_{1}$, s.t. $B \subset \tilde{L}_{1}$ and $A \cap \tilde{L}_{1}=\emptyset$.

(7) $\mathscr{L}_{1}$ separates $\mathscr{L}_{2}$ if

$\forall \tilde{\mathrm{L}}_{2}, \hat{\mathrm{L}}_{2} \in \mathscr{L}_{2}, \quad \tilde{\mathrm{L}}_{2} \cap \hat{\mathrm{L}}_{2}=\emptyset \Rightarrow$

$\exists \tilde{\mathrm{L}}_{1}, \hat{\mathrm{L}}_{1} \in \mathscr{L}_{1}$, s.t. $\tilde{\mathrm{L}}_{2} \subset \tilde{\mathrm{L}}_{1}, \hat{\mathrm{L}}_{2} \subset \hat{\mathrm{L}}_{1}$, and $\tilde{\mathrm{L}}_{1} \cap \hat{\mathrm{L}}_{1}=\emptyset$.

(8) $\mathscr{L}_{1}$ coseparates $\mathscr{L}_{2}$ if

$\forall \tilde{\mathrm{L}}_{2}, \hat{\mathrm{L}}_{2} \in \mathscr{L}_{2}, \quad \tilde{\mathrm{L}}_{2} \cap \hat{\mathrm{L}}_{2}=\emptyset \Rightarrow$

$\exists \tilde{\mathrm{L}}_{1}, \hat{\mathrm{L}}_{1} \in \mathscr{L}_{1}$, s.t. $\tilde{\mathrm{L}}_{2} \subset \tilde{\mathrm{L}}_{1}^{\prime}, \hat{\mathrm{L}}_{2} \subset \hat{\mathrm{L}}_{1}^{\prime}$, and $\tilde{\mathrm{L}}_{1} \cap \hat{\mathrm{L}}_{1}=\emptyset$.

(9) $\mathscr{L}_{2}$ coallocates $\mathscr{L}_{1}$ if

$$
\begin{aligned}
\forall \mathrm{L}_{1} \in \mathscr{L}_{1} \text { s.t. } & \mathrm{L}_{1} \subset \tilde{\mathrm{L}}_{2}^{\prime} \cup \hat{\mathrm{L}}_{2}^{\prime}, \text { where } \tilde{\mathrm{L}}_{2}, \hat{\mathrm{L}}_{2} \in \mathscr{L}_{2} \Rightarrow \\
& \exists \tilde{\mathrm{L}}_{1}, \hat{\mathrm{L}}_{1} \in \mathscr{L}_{1} \text { s.t. } \tilde{\mathrm{L}}_{1} \subset \tilde{\mathrm{L}}_{2}^{\prime}, \hat{\mathrm{L}}_{1} \subset \hat{\mathrm{L}}_{2}^{\prime}, \mathrm{L}_{1}=\tilde{\mathrm{L}}_{1} \cup \hat{\mathrm{L}}_{1} .
\end{aligned}
$$

\section{Definition $\mathbf{2 . 3}$}

A finitely additive (f.a.) measure $\mu$ is a finite nonnegative function defined on the algebra $A(\mathscr{L})$ generated by $\mathscr{L}$, such that (1) $\forall \mathrm{A} \in \mathrm{A}(\mathscr{L}), \mu(\mathrm{A}) \geq 0, \quad$ (2) $\mu(\emptyset)=0$, and (3) [finite additivity] $\forall \mathrm{A}, \mathrm{B} \in \mathrm{A}(\mathscr{L})$, $A \cap B=\emptyset \Rightarrow \mu(A \cup B)=\mu(A)+\mu(B)$.

A 0-1 measure $\mu$ is a two-valued finitely additive measure taking value either 0 or 1 .

Usually, we simply refer $\mu$ to as a measure on a lattice $\mathscr{L}$ to mean that $\mu$ is a finitely additive measure defined on the algebra $A(\mathscr{L})$.

A f.a. measure $\mu$ defined on the algebra $A(\mathscr{L})$ is

(1) $\mathscr{L}$-regular iff $\forall A \in A(\mathscr{L}), \mu(A)=\sup \left\{\mu(\mathrm{L})^{\prime}: \mathrm{L} \subset \mathrm{A}, \mathrm{L} \in \mathscr{L}\right\}$.

or, equivalently, $\mu(A)=\inf \left\{\mu\left(\hat{L}^{\prime}\right): \hat{L} \cdot \supset A, \hat{L} \in \mathscr{L}\right\}$.

(2) $\sigma$-smooth on $A(\mathscr{L})$, iff

$$
\forall A_{1}, A_{2}, \ldots \in A(\mathscr{L}), \quad A_{1} \supset A_{2} \supset \ldots \downarrow \emptyset,\left(A_{n} \downarrow \emptyset\right) \Rightarrow \mu\left(A_{n}\right) \rightarrow 0 \text { as } n \rightarrow \infty
$$

(3) $\sigma$-smooth on $\mathscr{L}$, iff

$$
\forall L_{1}, L_{2}, \ldots \in \mathscr{L}, \quad L_{1} \supset L_{2} \supset \ldots \downarrow \emptyset, \quad\left(L_{n} \downarrow \emptyset\right) \quad \Rightarrow \quad \mu\left(L_{n}\right) \rightarrow 0 \text { as } n \rightarrow \infty
$$

The following notations for the collections of measures on $A(\mathscr{L})$ will be used throughout :

$M(\mathscr{L}) \equiv\{\mu: \mu$ f.a. measure on $A(\mathscr{L})\}$

$M_{R}(\mathscr{L}) \equiv\{\mu \in M(\mathscr{L}): \mu \mathscr{L}$-regular $\}$

$M^{\sigma}(\mathscr{L}) \equiv\{\mu \in \mathrm{M}(\mathscr{L}): \mu$-smooth on $\mathrm{A}(\mathscr{L})\}$

$M_{\sigma}(\mathscr{L}) \equiv\{\mu \in \mathrm{M}(\mathscr{L}): \mu \quad \sigma$-smooth on $\mathscr{L}\}$ 


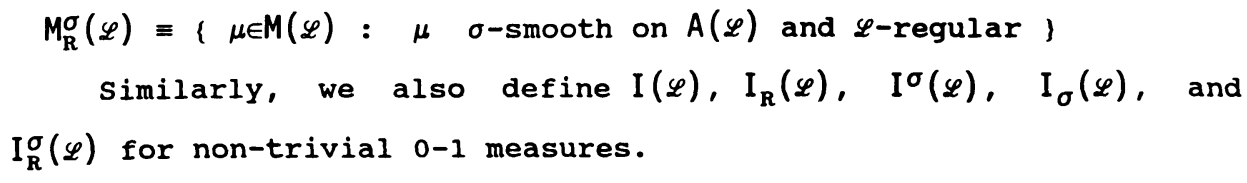

If $\mu$ is $\mathscr{L}$-regular, then $\sigma$-smoothness on $\mathscr{L}$ implies $\sigma$-smoothness on $A(\mathscr{L})$. Thus, $M_{R}^{\sigma}(\mathscr{L})=M_{R}(\mathscr{L}) \cap M_{\sigma}(\mathscr{L})=M_{R}(\mathscr{L}) \cap M^{\sigma}(\mathscr{L})$. since $A\left(\mathscr{L}^{\prime}\right)=A(\mathscr{L})$, we have $M\left(\mathscr{L}^{\prime}\right)=M(\mathscr{L})$ and $I\left(\mathscr{L}^{\prime}\right)=I(\mathscr{L})$. Furthermore, $\mu$ is $\sigma$-smooth on $A(\mathscr{L})\left(\mu \in M^{\sigma}(\mathscr{L})\right)$ iff $\mu$ is countably additive.

Let $\mu_{1}, \mu_{2} \in M(\mathscr{L})$. Define

$$
\begin{array}{lrr}
\mu_{1} \leq \mu_{2} \quad \text { if } & \forall \mathrm{A} \in \mathrm{A}(\mathscr{L}), \quad \mu_{1}(\mathrm{~A}) \leq \mu_{2}(\mathrm{~A}) \\
\mu_{1} \leq \mu_{2} \text { on } \mathscr{L}, & \text { if } \forall \mathrm{L} \in \mathscr{L}, & \mu_{1}(\mathrm{~L}) \leq \mu_{2}(\mathrm{~L}) \\
\mu_{1} \leq \mu_{2} \text { on } \mathscr{L}^{\prime}, & \text { if } \forall \mathrm{L} \in \mathscr{L}, & \mu_{1}\left(\mathrm{~L}^{\prime}\right) \leq \mu_{2}\left(\mathrm{~L}^{\prime}\right)
\end{array}
$$

\section{Definition 2.4}

Suppose $\mathscr{L}_{1} \subset \mathscr{L}_{2}$ are lattices of subsets of $X$ such that $\mu_{1} \in M\left(\mathscr{L}_{1}\right)$ and $\mu_{2} \in M\left(\mathscr{L}_{2}\right)$. Denote $\left.\mu_{2}\right|_{\mathscr{L}_{1}}$ (or simply $\mu_{2} \mid$ ) to mean the restriction of $\mu_{2}$ to $A\left(\mathscr{L}_{1}\right)$.

If $\mu_{1}=\mu_{2}$ on $A\left(\mathscr{L}_{1}\right)$, then $\mu_{2}$ is called a measure extension of $\mu_{1}$ from $\mathrm{A}\left(\mathscr{L}_{1}\right)$ to $\mathrm{A}\left(\mathscr{L}_{2}\right)$ (or, less precisely, from $\mathscr{L}_{1}$ to $\mathscr{L}_{2}$ ); and a regular measure extension, if $\mu_{2} \in M_{R}\left(\mathscr{L}_{2}\right)$.

If $\mu_{1} \leq \mu_{2} \mid$ on $\mathscr{L}_{1}$ and $\mu_{1}(X)=\mu_{2}(X)$, then $\mu_{2}$ is called a measure enlargement of $\mu_{1}$ from $A\left(\mathscr{L}_{1}\right)$ to $A\left(\mathscr{L}_{2}\right)$ (or, less precisely, from $\mathscr{L}_{1}$ to $\left.\mathscr{L}_{2}\right)$; and a regular measure enlargement, if $\mu_{2} \in M_{R}\left(\mathscr{L}_{2}\right)$.

\section{Definition $\mathbf{2 . 5}$}

A real-valued function $\mu_{*}: P(X) \rightarrow[0, \infty)$, is called a finitely superadditive inner measure, if

(1) $\mu_{*}(\emptyset)=0$

(2) [nondecreasing] $\forall \mathrm{A} \subset \mathrm{B} \subset \mathrm{X} \quad \Rightarrow \quad \mu_{*}(\mathrm{~A}) \leq \mu_{*}(\mathrm{~B})$, that is, $\mu_{*} \uparrow$

(3) [finite superadditivity] $\forall \mathrm{A}, \mathrm{B} \subset \mathrm{X}, \mathrm{A} \cap \mathrm{B}=\emptyset \Rightarrow \mu_{*}(\mathrm{~A} \cup \mathrm{B}) \geq \mu_{*}(\mathrm{~A})+\mu_{*}(\mathrm{~B})$

A real-valued function $\mu^{*}: P(X) \rightarrow[0, \infty)$, is called a finitely subadditive outer measure, if it satisfies (1), (2) and

(3') [finite subadditivity] $\forall \mathrm{A}, \mathrm{B} \subset \mathrm{X}, \mathrm{A} \cap \mathrm{B}=\emptyset \Rightarrow \mu^{*}(\mathrm{~A} \cup \mathrm{B}) \leq \mu^{*}(\mathrm{~A})+\mu^{*}(\mathrm{~B})$ Let $\mu^{*}$ be a finitely subadditive outer measure on $(X, \mathscr{L})$. A set $\mathrm{E} \subset X$ is said to be $\mu^{*}$-measurable, if

$$
\mu^{*}(\mathrm{~T})=\mu^{*}(\mathrm{~T} \cap \mathrm{E})+\mu^{*}\left(\mathrm{~T} \cap \mathrm{E}^{\prime}\right), \quad \forall \mathrm{T} \subset \mathrm{X} .
$$


We have the following theorem characterizing a normal lattice as a special case of the coallocation property :

\section{THEOREM 2.1}

$\mathscr{L}$ is normal $\Leftrightarrow \forall \mathrm{L} \in \mathscr{L}$ s.t. $\mathrm{L} \subset \mathrm{L}_{1}^{\prime} \cup \mathrm{L}_{2}^{\prime}$, where $\mathrm{L}_{1}, \mathrm{~L}_{2} \in \mathscr{L} \Rightarrow$

Proof:

$$
\exists \tilde{\mathrm{L}}_{1}, \tilde{\mathrm{L}}_{2} \in \mathscr{L} \text { s.t. } \mathrm{L}_{1} \subset \tilde{\mathrm{L}}_{1}, \mathrm{~L}_{2} \subset \tilde{\mathrm{L}}_{2}, L=\tilde{\mathrm{L}}_{1} \cup \tilde{\mathrm{L}}_{2} \text {. }
$$

Suppose $\mathrm{L}_{1}, \mathrm{~L}_{2} \in \mathscr{L}$ and $\mathrm{L}_{1} \cap \mathrm{L}_{2}=\emptyset . \therefore X=\mathrm{L}_{1}^{\prime} \cup \mathrm{L}_{2}^{\prime}$. Then by assumption, $\exists \tilde{\mathrm{L}}_{1}, \tilde{\mathrm{L}}_{2} \in \mathscr{L}$ such that $\tilde{\mathrm{L}}_{1} \subset \mathrm{L}_{1}^{\prime}, \tilde{\mathrm{L}}_{2} \subset \mathrm{L}_{2}^{\prime}$ and $X=\tilde{\mathrm{L}}_{1} \cup \tilde{\mathrm{L}}_{2}$ or $\tilde{\mathrm{L}}_{1}^{\prime} \cap \tilde{\mathrm{L}}_{2}^{\prime}=\emptyset$.

Thus, when $\mathrm{L}_{1} \cap \mathrm{L}_{2}=\emptyset, \mathrm{L}_{1} \subset \tilde{\mathrm{L}}_{1}^{\prime}, \mathrm{L}_{2} \subset \tilde{L}_{2}^{\prime}$, we have $\tilde{L}_{1}^{\prime} \cap \tilde{L}_{2}^{\prime}=\emptyset . \therefore \mathscr{L}$ is normal. $" \Rightarrow "$

Let $L \in \mathscr{L}$ and $L \subset L_{1}^{\prime} \cup L_{2}^{\prime}$ where $L_{1}, L_{2} \in \mathscr{L}$. Consider $L-L_{i}^{\prime}$ and $L-L_{2}^{\prime}$, $\left(L-L_{1}^{\prime}\right) \cap\left(L-L_{2}^{\prime}\right)=\left(L \cap L_{1}\right) \cap\left(L \cap L_{2}\right)=L \cap\left(L_{1}^{\prime} \cup L_{2}^{\prime}\right)^{\prime}=\emptyset . \quad \therefore L-L_{1}^{\prime}$ and $L-L_{2}^{\prime}$ are disjoint. By normality, $\exists \hat{L}_{1}, \hat{L}_{2} \in \mathscr{L}$, such that $\left(L-L_{i}^{\prime}\right) \subset \hat{L}_{1}^{\prime}$, $\left(\mathrm{L}-\mathrm{L}_{2}^{\prime}\right) \subset \hat{\mathrm{L}}_{2}^{\prime}$ and $\hat{\mathrm{L}}_{1}^{\prime} \cap \hat{\mathrm{L}}_{2}^{\prime}=\emptyset$, or $\hat{\mathrm{L}}_{1} \cup \hat{\mathrm{L}}_{2}=\mathrm{X}$. Define

$$
\hat{\mathrm{L}}_{1} \equiv\left(L-\hat{\mathrm{L}}_{1}^{\prime}\right)=\operatorname{L\cap } \hat{\mathrm{L}}_{1} \in \mathscr{L} \text { and } \tilde{\mathrm{L}}_{2} \equiv\left(L-\hat{\mathrm{L}}_{2}^{\prime}\right)=\operatorname{L\cap } \hat{\mathrm{L}}_{2} \in \mathscr{L}
$$

Then $\tilde{\mathrm{L}}_{1} \cup \tilde{\mathrm{L}}_{2}=\left(\operatorname{L\cap } \hat{\mathrm{L}}_{1}\right) \cup\left(\operatorname{L} \cap \hat{\mathrm{L}}_{2}\right)=\operatorname{L\cap }\left(\hat{\mathrm{L}}_{1} \cup \hat{\mathrm{L}}_{2}\right)=\operatorname{L} \cap X=L$. Now,

$$
\begin{aligned}
& L-L_{1}^{\prime} \subset \hat{L}_{1}^{\prime} \Rightarrow \hat{L}_{1} \subset L^{\prime} \cup L_{1}^{\prime} \text { and } L-L_{2}^{\prime} \subset \hat{L}_{2}^{\prime} \Rightarrow \hat{L}_{2} \subset L^{\prime} \cup L_{2}^{\prime} \\
& \therefore \quad \tilde{L}_{1}=\operatorname{Ln} \hat{L}_{1} \subset \operatorname{Ln}\left(L^{\prime} \cup L_{1}^{\prime}\right)=\left(L \cap L^{\prime}\right) \cup\left(L \cap L_{1}^{\prime}\right)=\left(L \cap L_{1}^{\prime}\right) \subset L_{1}^{\prime} \\
& \tilde{L}_{2}=\operatorname{L\cap } \hat{L}_{2} \subset L \cap\left(L^{\prime} \cup L_{i}^{\prime}\right)=\left(L \cap L^{\prime}\right) \cup\left(L \cap L_{2}^{\prime}\right)=\left(L \cap L_{2}^{\prime}\right) \subset L_{2}^{\prime}
\end{aligned}
$$

Thus, $\forall \mathrm{L} \in \mathscr{L}, L \subset L_{1}^{\prime} \cup L_{2}^{\prime}, \exists \tilde{L}_{1}, \tilde{L}_{2} \in \mathscr{L}$, s.t. $\tilde{L}_{1} \subset L_{1}^{\prime}, \tilde{L}_{2} \subset L_{2}^{\prime}$ and $L=\tilde{L}_{1} \cup \tilde{L}_{2}$.

The following results are obvious :

(1) $\mathscr{L}$ is normal $\Leftrightarrow \mathscr{L}$ coallocates itself $\Leftrightarrow \mathscr{L}$ coseparates itself.

(2) $\mathscr{L}_{1}$ separates $\mathscr{L}_{2} \Rightarrow \mathscr{L}_{1}$ semi-separates $\mathscr{L}_{2}$.

Furthermore, we have the following measure theoretic characterization of a normal lattice :

\section{THEOREM 2.2}

$\mathscr{L}$ is normal iff

$\forall \mu \in I(\mathscr{L})$, s.t. on $\mathscr{L}, \mu \leq \nu_{1} \in \mathrm{I}_{\mathrm{R}}(\mathscr{L}), \mu \leq \nu_{2} \in \mathrm{I}_{\mathrm{R}}(\mathscr{L}) \Rightarrow \nu_{1}=\nu_{2}$.

\section{THEOREM 2.3}

Suppose $\mathscr{L}_{1} \subset \mathscr{L}_{2}$. Then $\mathscr{L}_{1}$ coseparates $\mathscr{L}_{2} \Rightarrow \quad \mathscr{L}_{2}$ coallocates $\mathscr{L}_{1}$. Proof:

Suppose $\mathrm{L}_{1} \subset \tilde{L}_{2}^{\prime} \cup \hat{L}_{2}^{\prime}$, where $\mathrm{L}_{1} \in \mathscr{L}_{1}, \tilde{\mathrm{L}}_{2}, \hat{\mathrm{L}}_{2} \in \mathscr{L}$. Then, $\mathrm{L}_{1} \cap \tilde{\mathrm{L}}_{2}, \mathrm{~L}_{1} \cap \hat{\mathrm{L}}_{2} \in \mathscr{L}_{2} \supset \mathscr{L}_{1}$.

Now $\quad\left(\mathrm{L}_{1} \cap \tilde{\mathrm{L}}_{2}\right) \cap\left(\mathrm{L}_{1} \cap \hat{\mathrm{L}}_{2}\right)=\mathrm{L}_{1} \cap\left(\tilde{\mathrm{L}}_{2} \cap \hat{\mathrm{L}}_{2}\right)=\mathrm{L}_{1} \cap\left(\tilde{\mathrm{L}}_{2} \cup \hat{\mathrm{L}}_{2}^{\prime}\right)^{\prime}=\emptyset$.

$\mathscr{L}_{1}$ coseparates $\mathscr{L}_{2} \Rightarrow \exists \tilde{\mathrm{L}}_{1}, \hat{\mathrm{L}}_{1} \in \mathscr{L}_{1}$ s.t. $\tilde{\mathrm{L}}_{1} \cap \hat{\mathrm{L}}_{1}=\emptyset$, and 
$\mathrm{L}_{1} \cap \tilde{\mathrm{L}}_{2} \subset \tilde{\mathrm{L}}_{1}^{\prime}$ and $\mathbf{L}_{1} \cap \hat{\mathrm{L}}_{2} \subset \hat{\mathbf{L}}_{1}^{\prime} \cdot$

Define $\tilde{L}_{1}^{\circ} \equiv L_{1} \cap \tilde{L}_{1}$ and $\hat{\mathrm{L}}_{1} \equiv \mathrm{L}_{1} \cap \hat{\mathrm{L}}_{1}$, hence $\tilde{\mathrm{L}}_{1}, \hat{\mathrm{L}}_{1} \in \mathscr{L}_{1}$. And

$$
\tilde{L}_{1} \cup \hat{L}_{i}=\left(L_{1} \cap \tilde{L}_{1}\right) \cup\left(L_{1} \cap \hat{L}_{1}\right)=L_{1} \cap\left(\tilde{L}_{1} \cup \hat{L}_{1}\right)=L_{1} \cap\left(\tilde{L}_{1}^{\prime} \cap \hat{L}_{1}^{\prime}\right)^{\prime}=L_{1} \text {. }
$$

$\therefore \mathrm{L}_{1}=\tilde{\mathrm{L}}_{\mathbf{i}} \cup \hat{\mathrm{L}}_{\mathbf{i}}$. Now

$\tilde{L}_{1}=L_{1} \cap \tilde{L}_{1} \subset L_{1} \cap\left(L_{1} \cap \tilde{L}_{2}\right)^{\prime}=L_{1} \cap\left(L_{1}^{\prime} \cup \tilde{L}_{2}^{\prime}\right)=\left(L_{1} \cap L_{1}^{\prime}\right) \cup\left(L_{1} \cap \tilde{L}_{2}^{\prime}\right)$

$=\mathrm{L}_{1} \cap \tilde{L}_{2}^{\prime} \subset \tilde{L}_{2}^{\prime}$.

Thus, $\tilde{L}_{1} \subset \tilde{L}_{2}$. Similarly, $\hat{L}_{i} \subset \hat{L}_{2}$. Hence $\mathscr{L}_{2}$ coallocates $\mathscr{L}_{1}$.

\section{THEOREM 2.4}

$$
\mathscr{L} \text { countably paracompact } \Rightarrow M_{\sigma}\left(\mathscr{L}^{1}\right) \subset M_{\sigma}(\mathscr{L})
$$

Proof:

$$
\begin{aligned}
& \text { Suppose } \forall n, L_{n} \in \mathscr{L}, L_{n} \downarrow \emptyset \\
& \mathscr{L} \text { c.p. } \Rightarrow \quad \exists \tilde{L}_{n} \in \mathscr{L}, \text { s.t. } L_{n} \subset \tilde{L}_{n}^{\prime}, \tilde{L}_{n}^{\prime} \downarrow \emptyset \\
& \mu \in M_{\sigma}(\mathscr{L}) \quad \Leftrightarrow \mu\left(\tilde{L}_{n}^{\prime}\right) \rightarrow 0 \text { as } \tilde{L}_{n}^{\prime} \downarrow \emptyset \\
& \therefore \mu\left(L_{n}\right) \leq \mu\left(\tilde{L}_{n}^{\prime}\right) \rightarrow 0, \quad \Rightarrow \mu\left(L_{n}\right) \rightarrow 0 \\
& \text { Now } L_{n} \downarrow \emptyset, \mu\left(L_{n}\right) \rightarrow 0 \quad \therefore \mu \in M_{\sigma}(\mathscr{L}) \text { Hence, } M_{\sigma}(\mathscr{L}) \subset M_{\sigma}(\mathscr{L}) .
\end{aligned}
$$

\section{MEASURES ON COALLOCATION LATTICES}

In this section we extend some of the work of [8] and [2] on the unique extendability of a measure $\mu \in M_{R}\left(\mathscr{L}_{1}\right)$ to a measure $\nu \in M_{R}\left(\mathscr{L}_{2}\right)$ where $\mathscr{L}_{1}$ and $\mathscr{L}_{2}$ are lattices of subsets of $X$. We note that it is not always necessary to assume that $\mathscr{L}_{1} \subset \mathscr{L}_{2}$ nor that $\mathrm{X}$ belongs to the lattices in order for the main results of the coallocation theorem to hold (see Theorem 3.1). We first define two functions which form an inner-outer measure pair.

\section{Definition 3.1}

Suppose $\mathscr{L}_{1}$ and $\mathscr{L}_{2}$ are lattices of subsets of $X$ and $\mu \in M\left(\mathscr{L}_{1}\right)$. For all ECX, define and

$$
\begin{aligned}
& \mu_{.}(E) \equiv \sup \left(\mu\left(L_{1}\right): E \supset L_{1}, L_{1} \in \mathscr{L}_{1}\right) \\
& \mu^{\wedge}(E)=\inf \left(\mu_{.}\left(L_{2}^{\prime}\right): E C L_{2}^{\prime}, L_{2} \in \mathscr{L}_{2}\right\}
\end{aligned}
$$

We have the following :

\section{THEOREM 3.1 [Coallocation theorem]}

Let $\mathscr{L}_{1}$ and $\mathscr{L}_{2}$ be lattices of subsets of $X \neq \emptyset$. Suppose $\mu \in M\left(\mathscr{Q}_{1}\right)$. We have 
(1) $\mu$. is a finitely superadditive inner measure

(2) $\mathscr{L}_{2}$ coallocates $\mathscr{L}_{1} \quad \Rightarrow \mu$. is finitely additive on $\mathscr{L}_{2}^{\prime}$

(3) $\mathscr{L}_{2}$ coallocates $\mathscr{L}_{1} \Rightarrow \mu^{\wedge}$ is a finitely subadditive outer measure

(4) $\mu^{\wedge}=\mu$. on $\mathscr{L}_{2}^{\prime}$

In particular, if $X \in \mathscr{L}_{1}$ and $\emptyset \in \mathscr{L}_{2}$, then $\mu^{\wedge}(X)=\mu .(X)=\mu(X)$

(5) $[\mathrm{a}] \mu \leq \mu^{\wedge}$ on $\mathscr{L}_{1}$

[b] $\mathscr{L}_{1} \subset \mathscr{L}_{2}$ and $\mu \in M_{R}\left(\mathscr{L}_{1}\right) \Rightarrow \mu .=\mu$ on $\mathscr{L}_{1}^{\prime} ; \mu^{\wedge}=\mu$ on $\mathscr{L}_{1}$

(6) Suppose $\mathscr{L}_{2}$ coallocates $\mathscr{L}_{1}, \quad \mathrm{E} \subset \mathrm{X}$ is $\mu^{*}$-measurable

$\Leftrightarrow \quad \forall L_{2} \in \mathscr{L}_{2}, \quad \mu^{\wedge}\left(L_{2}^{\prime}\right) \geq \mu^{\wedge}\left(L_{2}^{\prime} \cap E\right)+\mu^{\wedge}\left(L_{2}^{\prime} \cap E^{\prime}\right)$

(7) Suppose $\mathscr{L}_{2}$ coallocates $\mathscr{L}_{1}$.

If either [a] $\mathscr{L}_{1} \subset \mathscr{L}_{2}$

then or [b] $\mathscr{L}_{2}$ semi-separates $\mathscr{L}_{1}$

[1०] every element of $\mathscr{L}_{2}^{\prime}$ is $\mu^{*}$-measurable

$\left[2^{\circ}\right] \mu^{\wedge} \mid \mathscr{L}_{2}$ is a finitely additive measure on $A\left(\mathscr{L}_{2}\right)$

$\left[3^{\circ}\right] \mu^{\wedge}$ is $\mathscr{L}_{1}$-regular on $\mathscr{L}_{2}^{\prime}$

$\left[4^{\circ}\right] \mu^{\wedge} \in M_{R}\left(\mathscr{L}_{2}\right)$.

Proof:

(1) The proof is standard and is therefore omitted.

(2) Let $\mathrm{A}_{2}, \mathrm{~B}_{2} \in \mathscr{L}_{2}$, and $\mathrm{L}_{1} \in \mathscr{L}$ s.t. $\mathrm{L}_{1} \subset \mathrm{A}_{2}^{\prime} \cup \mathrm{B}_{2}^{\prime} \in \mathscr{L}_{2}^{\prime}$

$\mathscr{L}_{2}$ coallocates $\mathscr{L}_{1} \Rightarrow$

$\exists A_{1}, B_{1} \in \mathscr{L}_{1}$ s.t. $A_{1} \subset A_{2}^{\prime}, B_{1} \subset B_{2}^{\prime}$, and $L_{1}=A_{1} \cup B_{1}$

$\therefore \mu\left(\mathrm{L}_{1}\right)=\mu\left(\mathrm{A}_{1} \cup \mathrm{B}_{1}\right)$

$\leq \mu\left(A_{1}\right)+\mu\left(B_{1}\right)$

$\leq \sup \left\{\mu\left(A_{1}\right): A_{2}^{\prime} \supset A_{1}\right\}+\sup \left\{\mu\left(B_{1}\right): B_{2}^{\prime} \supset B_{1}\right\}$

$\equiv \mu_{.}\left(A_{2}^{\prime}\right)+\mu_{.}\left(B_{2}^{\prime}\right)$

Taking sup on the left hand side,

$\sup \left\{\mu\left(L_{1}\right): L_{1} \subset A_{2}^{\prime} \cup B_{2}^{\prime}\right\} \leq \mu .\left(A_{2}^{\prime}\right)+\mu_{.}\left(B_{2}^{\prime}\right)$

$\therefore \mu .\left(A_{2}^{\prime} \cup B_{2}^{\prime}\right) \leq \mu .\left(A_{2}^{\prime}\right)+\mu .\left(B_{2}^{\prime}\right) \Rightarrow$

$\mu$. is finitely subadditive on $\mathscr{L}_{2}$. Together with (1), $\mu$. is finitely additive on $\mathscr{L}_{\dot{2}}^{\prime}$.

(3) The proof is also standard and is omitted.

(4) Let $\mathrm{L}_{2} \in \mathscr{L}_{2}$.

$\mu^{\wedge}\left(L_{2}^{\prime}\right)=\inf \left(\mu_{0}\left(\tilde{L}_{2}^{\prime}\right): L_{2}^{\prime} \subset \tilde{L}_{2}^{\prime}, \tilde{L}_{2} \in \mathscr{L}_{2}\right)$

$\Rightarrow \mu^{\wedge}\left(L_{2}^{\prime}\right) \leq \mu .\left(L_{2}^{\prime}\right)$ 
Now if $A_{2}^{\prime} \in \mathscr{L}_{2}^{\prime}$ and $L_{2}^{\prime} \subset A_{2}^{\prime}$, then by monotonicity of $\mu$. ,

$\mu_{\text {. }}\left(\mathrm{L}_{2}^{\prime}\right) \leq \mu_{\text {. }}\left(\mathrm{A}_{2}{ }^{\prime}\right)$

$\Rightarrow \mu_{.}\left(L_{2}^{\prime}\right) \leq \inf \left(\mu_{.}\left(A_{2}^{\prime}\right): L_{2}^{\prime} \subset A_{2}^{\prime}, A_{2} \in \mathscr{L}_{2}\right\}=\mu^{\wedge}\left(L_{2}^{\prime}\right)$

[i] and [ii] $\Rightarrow \mu^{\wedge}=\mu$. on $\mathscr{L}_{2}^{\prime}$.

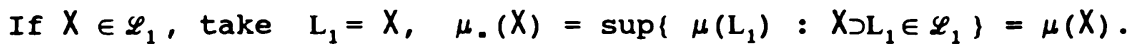

If $\emptyset \in \mathscr{L}_{2}, X=\emptyset, \in \mathscr{L}_{2}^{\prime}$ and $\mu^{\wedge}=\mu$. on $\mathscr{L}_{2}^{\prime} \Rightarrow \mu^{\wedge}(X)=\mu$. (X)

Consequently, $\mu^{\wedge}(X)=\mu .(X)=\mu(X)$.

(5)[a] Let $\mathrm{L}_{1} \in \mathscr{L}_{1}$ and $\mathrm{A}_{2} \in \mathscr{L}_{2}$, s.t. $\mathrm{L}_{1} \subset \mathrm{A}_{2}^{\prime}$

$\mu .\left(A_{2}^{\prime}\right)=\sup \left(\mu\left(L_{1}\right): A_{2}^{\prime} \supset L_{1} \in \mathscr{L}_{1}\right) \geq \mu\left(L_{1}\right)$

Taking inf, inf $\left(\mu_{0}\left(A_{2}^{\prime}\right): L_{1} \subset A_{2}^{\prime}, A_{2} \in \mathscr{L}_{2}\right) \geq \mu\left(L_{1}\right)$

i.e. $\mu^{\wedge}\left(L_{1}\right) \geq \mu\left(L_{1}\right)$. or, $\mu \leq \mu^{\wedge}$ on $\mathscr{L}_{1}$.

(5)[b] Suppose $\mathscr{L}_{1} \subset \mathscr{L}_{2}$, then $\mu^{\wedge}=\mu$. on $\mathscr{L}_{2}^{\prime} \Rightarrow \mu^{\wedge}=\mu$. on $\mathscr{L}_{1}^{\prime}$

$\therefore$ if $\tilde{\mathrm{L}}_{1} \in \mathscr{L}_{1}$, then $\mu^{\wedge}\left(\tilde{\mathrm{L}}_{1}^{\prime}\right)=\mu$. $\left(\tilde{\mathrm{L}}_{1}^{\prime}\right)$

Suppose $\mathrm{L}_{1} \subset \mathrm{A}_{2}^{\prime} \subset \mathrm{A}_{1}^{\prime}$, where $\mathrm{A}_{1} \in \mathscr{L}_{1}, \mathrm{~A}_{2} \in \mathscr{L}_{2}, \mathrm{~A}_{1} \subset \mathrm{A}_{2}$

$\mu .\left(A_{1}^{\prime}\right)=\sup \left\{\mu\left(\tilde{L}_{1}\right): A_{1} \subset \tilde{L}_{1} \in \mathscr{L}_{1}\right\}$

But $\mu \in M_{R}\left(\mathscr{L}_{1}\right) \Rightarrow \mu\left(\mathrm{A}_{1}^{\prime}\right)=\sup \left\{\mu\left(\tilde{L}_{1}\right): \tilde{L}_{1} \in \mathscr{L}_{1}\right\}$ whenever $A_{1} \subset \tilde{L}_{1}$

$\therefore \mu .\left(\mathrm{A}_{1}^{\prime}\right)=\mu\left(\mathrm{A}_{1}^{\prime}\right) \quad \forall \mathrm{A}_{1}^{\prime} \in \mathscr{L}_{1}^{\prime}$, hence $\mu_{.}=\mu$ on $\mathscr{L}_{1}^{\prime}$.

Now, $\mu^{\wedge}\left(L_{1}\right)=\inf \left\{\mu_{.}\left(A_{2}^{\prime}\right): L_{1} \subset A_{2}^{\prime}, A_{2} \in \mathscr{L}_{2}\right\}$

$$
\begin{array}{ll}
\leq \inf \left(\mu .\left(A_{1}^{\prime}\right): L_{1} \subset A_{1}^{\prime}, A_{1} \in \mathscr{L}_{1}\right\} & \left(\because A_{2}^{\prime} \subset A_{1}^{\prime}\right) \\
=\inf \left(\mu\left(A_{1}^{\prime}\right): L_{1} \subset A_{1}^{\prime}, A_{1} \in \mathscr{L}_{1}\right) & \left(\because \mu .=\mu \text { on } \mathscr{L}_{1}^{\prime}\right) \\
=\mu\left(L_{1}\right) & \left(\because \mu \in M_{R}\left(\mathscr{L}_{1}\right)\right)
\end{array}
$$

$\therefore \mu^{\wedge} \leq \mu$ on $\mathscr{L}_{1}$, and $(5)[\mathrm{a}] \Rightarrow \mu \leq \mu^{\wedge}$ on $\mathscr{L}_{1}, \therefore \mu^{\wedge}=\mu$ on $\mathscr{L}_{1}$.

(6) $" \leftleftarrows n \quad$ Suppose $\forall A_{2} \in \mathscr{L}_{2}$, we have $\forall E \subset X$,

$$
\mu^{\wedge}\left(A_{2}^{\prime}\right) \geq \mu^{\wedge}\left(A_{2}^{\prime} \cap E\right)+\mu^{\wedge}\left(A_{2}^{\prime} \cap E^{\prime}\right)
$$

Suppose $\mathrm{T} \subset \mathrm{X}$, s.t. $\mathrm{T} \subset \mathrm{A}_{2}^{\prime}, \mathrm{A}_{2} \in \mathscr{L}_{2}$

$$
\mu^{\wedge}(T)=\inf \left(\mu_{.}\left(A_{2}^{\prime}\right): T \subset A_{2}^{\prime}, A_{2} \in \mathscr{L}_{2}\right\}
$$

Now $(4) \Rightarrow$

$$
\begin{array}{rlrl}
\mu .\left(A_{2}^{\prime}\right) & =\mu^{\wedge}\left(A_{2}^{\prime}\right) \\
& \geq \mu^{\wedge}\left(A_{2}^{\prime} \cap E\right)+\mu^{\wedge}\left(A_{2}^{\prime} \cap E^{\prime}\right) & \quad(\text { by assumption }) \\
& \geq \mu^{\wedge}(T \cap E)+\mu^{\wedge}\left(T \cap E^{\prime}\right) & \left(\because T \subset A_{2}^{\prime}, \mu^{\wedge} \uparrow\right)
\end{array}
$$

Taking inf,

$$
\mu^{\wedge}(T) \geq \mu^{\wedge}(\mathrm{T} \cap E)+\mu^{\wedge}\left(\mathrm{T} \cap E^{\prime}\right)
$$

$\mu^{\wedge}$ is finitely subadditive $\Rightarrow$

$\mu^{\wedge}(T)=\mu^{\wedge}\left(\mathrm{T} \cap\left(E \cup E^{\prime}\right)\right) \leq \mu^{\wedge}(\mathrm{T} \cap E)+\mu^{\wedge}\left(\mathrm{T} \cap E^{\prime}\right)$ 
[iii] and [iv] $\Rightarrow \mu^{\wedge}(T)=\mu^{\wedge}(T \cap E)+\mu^{\wedge}\left(T \cap E^{\prime}\right) \quad \forall T \subset X$, which is the definition of $E$ to be $\mu^{\wedge}$-measurable.

(6) " $\Rightarrow "$ By the definition of $E$ to be $\mu^{\wedge}$-measurable, we have $\mu^{\wedge}(T)=\mu^{\wedge}(T \cap E)+\mu^{\wedge}\left(T \cap E^{\prime}\right) \quad \forall T \subset X$,

But $\mu^{\wedge}$ is finitely subadditive, the above is equivalent to $\mu^{\wedge}(T) \geq \mu^{\wedge}(T \cap E)+\mu^{\wedge}\left(T \cap E^{\prime}\right) \quad \forall T \subset X$,

In particular, take $T=L_{2}^{\prime} \in \mathscr{L}_{2}^{\prime}$, we have

$\mu^{\wedge}\left(L_{2}^{\prime}\right) \geq \mu^{\wedge}\left(L_{2}^{\prime} \cap E\right)+\mu^{\wedge}\left(L_{2}^{\prime} \cap E^{\prime}\right) \quad \forall L_{2} \in \mathscr{L}_{2}$.

(7) Suppose $\mathscr{L}_{2}$ coallocates $\mathscr{L}_{1}$.

Let $L_{2}^{\prime} \in \mathscr{L}_{2}^{\prime}$. To prove that $L_{2}^{\prime}$ is $\mu^{\wedge}$-measurable, we have to show, by (6), that

$$
\begin{aligned}
\mu^{\wedge}\left(\mathrm{A}_{2}^{\prime}\right) & \geq \mu^{\wedge}\left(\mathrm{A}_{2}^{\prime} \cap L_{2}^{\prime}\right)+\mu^{\wedge}\left(\mathrm{A}_{2}^{\prime} \cap L_{2}\right) \quad \forall \mathrm{A}_{2} \in \mathscr{L}_{2} \cdot \\
\forall \mathrm{A}_{2} \in \mathscr{L}_{2}, \text { let } & P, Q \in \mathscr{L}_{1} \quad\left(\therefore \mathrm{P} \cup \mathrm{Q} \in \mathscr{L}_{1}\right) \text { s.t. } \\
& P \subset \mathrm{A}_{2}^{\prime} \cap L_{2}^{\prime} \quad \text { and } \mathrm{Q} \subset \mathrm{A}_{2}^{\prime} \cap \mathrm{P}^{\prime}
\end{aligned}
$$

Thus, $\quad P \subset A_{2}^{\prime}$ and $Q \subset A_{2}^{\prime}$

Now, $\quad P \cup Q \subset\left(A_{2}^{\prime} \cap L_{2}^{\prime}\right) \cup\left(A_{2}^{\prime} \cap P^{\prime}\right) \subset A_{2}^{\prime}$

and $\quad P \cap Q \subset P \cap\left(A_{2}^{\prime} \cap P^{\prime}\right)=\emptyset$

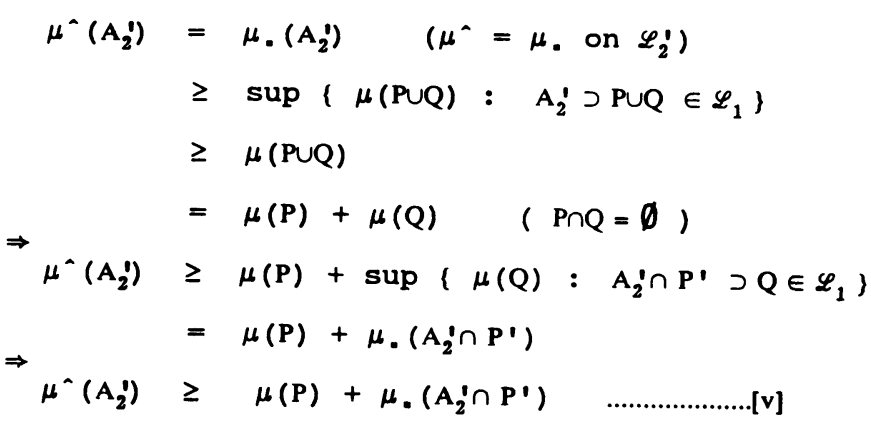

(7)[a] : Suppose $\mathscr{L}_{1} \subset \mathscr{L}_{2}$.

$$
\begin{aligned}
& \Rightarrow \quad \text { If } \mathscr{L}_{1} \subset \mathscr{L}_{2}, \text { then } \mathrm{P} \in \mathscr{L}_{1} \Rightarrow \mathrm{P} \in \mathscr{L}_{2} \quad \therefore \mathrm{A}_{2} \cup \mathrm{P} \in \mathscr{L}_{2} \\
& \mathrm{~A}_{2}^{\prime} \cap \mathrm{P}^{\prime}=\left(\mathrm{A}_{2} \cup \mathrm{P}\right)^{\prime} \in \mathscr{L}_{2}^{\prime} \text {, and } \mu^{\wedge}=\mu \text {. on } \mathscr{L}_{2}^{\prime}, \therefore[\mathrm{v}] \Rightarrow \\
& \mu^{\wedge}\left(\mathrm{A}_{2}^{\prime}\right) \geq \mu(\mathrm{P})+\mu^{\wedge}\left(\mathrm{A}_{2}^{\prime} \cap \mathrm{P}^{\prime}\right) \\
& \Rightarrow \mu(\mathrm{P})+\mu^{\wedge}\left(\mathrm{A}_{2}^{\prime} \cap \mathrm{L}_{2}\right) \quad\left(\mathrm{P} \subset \mathrm{L}_{2}^{\prime}\right) \geq \mu^{\wedge}\left(\mathrm{A}_{2}^{\prime} \cap \mathrm{L}_{2}\right) \\
& \mu^{\wedge}\left(\mathrm{A}_{2}^{\prime}\right)\left.\geq \sup \left(\mu(\mathrm{P}): \mathrm{A}_{2}^{\prime} \cap \mathrm{L}_{2}^{\prime} \supset \mathrm{P} \in \mathscr{L}_{1}\right\}+\mu^{\prime}\right) \\
& \equiv \mu_{.}\left(\mathrm{A}_{2}^{\prime} \cap \mathrm{L}_{2}^{\prime}\right)+\mu^{\wedge}\left(\mathrm{A}_{2}^{\prime} \cap \mathrm{L}_{2}\right) \\
&=\mu^{\wedge}\left(\mathrm{A}_{2}^{\prime} \cap \mathrm{L}_{2}^{\prime}\right)+\mu^{\wedge}\left(\mathrm{A}_{2}^{\prime} \cap \mathrm{L}_{2}\right) \quad \forall \mathrm{A}_{2} \in \mathscr{L}_{2}\left(\mu^{\wedge}=\mu \text {. on } \mathscr{L}_{2}^{\prime}\right)
\end{aligned}
$$

We conclude, from (6), that every element of $\mathscr{L}_{2}^{\prime}$ is $\mu^{\wedge}$-measurable.

$\therefore A\left(\mathscr{L}_{2}\right)=A\left(\mathscr{L}_{2}^{\prime}\right) \subset\left\{\mu^{\wedge}\right.$-measurable sets $\}$. By a standard carathéodory 
argument, $\mu^{-} \mid \mathscr{L}_{2}$ is a finitely additive measure on $A\left(\mathscr{L}_{2}\right)$.

Suppose $\mathrm{L}_{2} \in \mathscr{L}_{2}$,

$$
\begin{array}{rlr}
\mu^{\wedge}\left(L_{2}^{\prime}\right) & =\mu_{0}\left(L_{2}^{\prime}\right) & (\text { by }(4)) \\
& =\sup \left(\mu\left(L_{1}\right): L_{1} \subset L_{2}^{\prime}, L_{1} \in \mathscr{L}_{1}\right) \\
& \leq \sup \left(\mu^{\wedge}\left(L_{1}\right): L_{1} \subset L_{2}^{\prime}, L_{1} \in \mathscr{L}_{1}\right\}
\end{array}
$$

But $L_{1} \subset L_{2}^{\prime} \Rightarrow \mu^{\wedge}\left(L_{1}\right) \leq \mu^{\wedge}\left(L_{2}^{\prime}\right)$, taking sup $\Rightarrow$

Hence,

$$
\sup \left\{\mu^{\wedge}\left(L_{1}\right): L_{1} \subset L_{2}^{\prime}, L_{1} \in \mathscr{L}_{1}\right\} \leq \mu^{\wedge}\left(L_{2}^{\prime}\right)
$$

$$
\mu^{\wedge}\left(L_{2}^{\prime}\right)=\sup \left\{\mu^{\wedge}\left(L_{1}\right): L_{1} \subset L_{2}^{\prime}, L_{1} \in \mathscr{L}_{1}\right\}
$$

which means that $\mu^{-}$is $\mathscr{L}_{1}$-regular on $\mathscr{L}_{2}^{\prime}$. Since $\mathscr{L}_{1} \subset \mathscr{L}_{2}, \mu^{-}$is also $\mathscr{L}_{2}$-regular on $\mathscr{L}_{2}^{\prime}$. Now any element of $A\left(\mathscr{L}_{2}\right)$ is, of the form

$$
\bigcup_{i=1}^{n}\left(A_{i} \cap B_{i}^{\prime}\right) \quad A_{i}, B_{i} \in \mathscr{L}_{2}
$$

Consequently, $\mu^{\wedge} \in M_{R}\left(\mathscr{L}_{2}\right)$.

(7)[b] : Suppose $\mathscr{L}_{2}$ semi-separates $\mathscr{L}_{1}$.

Now $P \subset A_{2}^{\prime} \cap L_{2}^{\prime} \Rightarrow P \subset L_{2}^{\prime} \quad \Rightarrow \quad P \cap L_{2}=\emptyset$

and $\mathrm{P} \in \mathscr{L}_{1}, \mathrm{~L}_{2} \in \mathscr{L}_{2}$.

$\mathscr{L}_{2}$ semi-separates $\mathscr{L}_{1} \Rightarrow$

$\exists \tilde{L}_{2} \in \mathscr{L}_{2}$ s.t. $P \subset \tilde{L}_{2} \subset L_{2}^{\prime} \cdot \quad \therefore P^{\prime} \supset \tilde{L}_{2}^{\prime} \supset L_{2} \Rightarrow A_{2}^{\prime} \cap P^{\prime} \supset A_{2}^{\prime} \cap \tilde{L}_{2}^{\prime}$

From [v],

$$
\begin{array}{rlr}
\mu^{\wedge}\left(A_{2}^{\prime}\right) & \geq \mu(P)+\mu \cdot\left(A_{2}^{\prime} \cap P^{\prime}\right) & \\
& \geq \mu(P)+\mu \cdot\left(A_{2}^{\prime} \cap \dot{L}_{2}^{\prime}\right) & \\
& =\mu(P)+\mu^{\wedge}\left(A_{2}^{\prime} \cap \tilde{L}_{2}^{\prime}\right) & (\text { by }(4)) \\
& \geq \mu(P)+\mu^{\wedge}\left(A_{2}^{\prime} \cap L_{2}\right) & \left(\tilde{L}_{2}^{\prime} \supset L_{2}\right) \\
\Rightarrow \mu^{\wedge}\left(A_{2}^{\prime}\right) & \geq \sup \left(\mu(P): A_{2}^{\prime} \cap L_{2}^{\prime} \supset P \in \mathscr{L}_{1}\right)+\mu^{\wedge}\left(A_{2}^{\prime} \cap L_{2}\right) \\
& =\mu \cdot\left(A_{2}^{\prime} \cap L_{2}^{\prime}\right)+\mu^{\wedge}\left(A_{2}^{\prime} \cap L_{2}\right) & \\
& =\mu^{\wedge}\left(A_{2}^{\prime} \cap L_{2}^{\prime}\right)+\mu^{\wedge}\left(A_{2}^{\prime} \cap L_{2}\right), \forall A_{2} \in \mathscr{L}_{2}\left(\mu^{\wedge}=\mu . \text { on } \mathscr{L}_{2}^{\prime}\right)
\end{array}
$$

We conclude, from (6), that every element of $\mathscr{L}_{2}^{\prime}$ is $\mu^{\wedge}$-measurable. $\therefore A\left(\mathscr{L}_{2}\right)=A\left(\mathscr{L}_{2}^{\prime}\right) \subset\left\{\mu^{\wedge}\right.$-measurable sets $\}$. By a standard Carathéodory argument, $\mu^{\wedge} \mid \mathscr{L}_{2}$ is a finitely additive measure on $A\left(\mathscr{L}_{2}\right)$.

$$
\begin{aligned}
& \text { Let } \mathrm{L}_{2} \in \mathscr{L}_{2} \text {. Suppose } \mathrm{L}_{1} \subset \mathrm{L}_{2}^{\prime} \mathrm{L}_{1} \in \mathscr{L}_{1} \cdot \mathscr{L}_{2} \text { semi-separates } \mathscr{L}_{1} \\
& \Rightarrow \quad \begin{aligned}
& \exists \tilde{\mathrm{L}}_{2} \in \mathscr{L}_{2} \text { s.t. } \\
& \mu\left(\mathrm{L}_{1}\right)=\mathrm{L}_{1} \subset \tilde{\mathrm{L}}_{2} \subset \mathrm{L}_{2}^{\prime}\left(\mathrm{L}_{1}\right) \\
& \leq \mu^{\wedge}\left(\tilde{\mathrm{L}}_{2}\right) \leq \mu^{\wedge}\left(\mathrm{L}_{2}^{\prime}\right)
\end{aligned} \\
& \therefore \quad \mu^{\wedge}=\mu^{\wedge} \text { on } \mathscr{L}_{1}, \\
& \mu\left(\mathrm{L}_{1}\right) \leq \mu^{\wedge}\left(\mathrm{L}_{2}^{\prime}\right)
\end{aligned}
$$

Taking sup,

$\sup \left\{\mu\left(L_{1}\right): L_{1} \subset L_{2}^{\prime}, L_{1} \in \mathscr{L}_{1}\right\} \leq \sup \left\{\mu^{\wedge}\left(\tilde{L}_{2}\right): \tilde{L}_{2} \subset L_{2}^{\prime}, \tilde{L}_{2} \in \mathscr{L}_{2}\right\} \leq \mu^{\wedge}\left(L_{2}^{\prime}\right)$ 
But $\mu^{\wedge}\left(L_{2}^{\prime}\right)=\sup \left\{\mu\left(L_{1}\right) \cdot L_{1} \subset L_{2}^{\prime}, L_{1} \in \mathscr{L}_{1}\right\} \quad$ Hence,

$\mu^{\wedge}\left(\mathrm{L}_{2}^{\prime}\right)=\sup \left\{\mu\left(\mathrm{L}_{1}\right): \mathrm{L}_{1} \subset \hat{\mathbf{L}}_{2}, \mathbf{L}_{1} \in \mathscr{L}_{1}\right\}=\sup \left\{\mu^{\wedge}\left(\hat{\mathrm{L}}_{2}\right): \hat{\mathbf{L}}_{2} \subset \mathbf{L}_{2}^{\prime}, \hat{\mathrm{L}}_{2} \in \mathscr{L}_{2}\right\}$

$\therefore \quad \mu^{\wedge} \quad$ is $\mathscr{L}_{1}$-regular on $\mathscr{L}_{2}^{\prime}$ and $\mathscr{L}_{2}$-regular on $\mathscr{L}_{2}^{\prime}$, and consequently, $\mu^{\wedge} \in M_{R}\left(\mathscr{L}_{2}\right)$.

Note: If $\mathscr{L}_{1} \subset \mathscr{L}_{2}$, then $\mathscr{L}_{2}$ trivially semi-separates $\mathscr{L}_{1},(7)[\mathrm{a}] \Rightarrow(7)[\mathrm{b}]$

\section{Corollary 3.1}

Suppose $\mathscr{L}_{1}=\mathscr{L}_{2}=\mathscr{L}, \mathrm{X} \in \mathscr{L}$, and $\mathscr{L}$ coallocates itself, ( $\mathscr{L}$ is normal).

then

Proof:

$$
\text { (1) } \mu^{\wedge} \text { is finitely additive and } \mu^{\wedge}(X)=\mu .(X)=\mu(X)
$$$$
\text { (2) } \left.\mu^{\wedge}(\mathrm{L})+\mu . \mathrm{L}^{\prime}\right)=\mu(\mathrm{X}) \quad \forall \mathrm{L} \in \mathscr{L}
$$

(1) Direct consequences of Theorem 3.1.

(2) From Theorem 3.1(4), $\mu^{\wedge}=\mu$. on $\mathscr{L}_{2}^{\prime}=\mathscr{L}^{\prime} . \quad \therefore \mu^{\wedge}\left(\mathrm{L}^{\prime}\right)=\mu$. (L') $\forall \mathrm{L} \in \mathscr{L}$ Now $\mu^{\wedge}$ is finitely additive,

$$
\mu^{\wedge}(X)=\mu^{\wedge}\left(L \cup L^{\prime}\right)=\mu^{\wedge}(L)+\mu^{\wedge}\left(L^{\prime}\right)=\mu^{\wedge}(L)+\mu .\left(L^{\prime}\right)
$$

But $\mu^{\wedge}(X)=\mu_{0}(X)=\mu(X), \quad \therefore \mu^{\wedge}(L)+\mu_{0}\left(L^{\prime}\right)=\mu(X)$.

The coallocation theorem leads to the following direct consequences whose proofs are omitted.

\section{THEOREM 3.2 [Regular measure extension on coallocation lattices]}

Suppose $\mathscr{L}_{1} \subset \mathscr{L}_{2}$ and $\mu \in \mathrm{M}_{\mathrm{R}}\left(\mathscr{L}_{1}\right)$. If $\mathscr{L}_{2}$ coallocates $\mathscr{L}_{1}$, then there exists a unique $\nu \in M_{R}\left(\mathscr{L}_{2}\right)$, s.t. on $\mathscr{L}_{1}, \mu=\left.\nu\right|_{\mathscr{L}_{1}} \in \mathrm{M}_{\mathrm{R}}\left(\mathscr{L}_{1}\right)$. Furthermore, $\nu$ is $\mathscr{L}_{1}$-regular on $\mathscr{L}_{2}^{\prime}$. Note that $\nu=\dot{\mu}^{\wedge} \mid \mathscr{L}_{2}$.

THEOREM 3.3 [Regular measure enlargement on coallocation lattices]

Suppose $\mathscr{L}_{1} \subset \mathscr{L}_{2}$ and $\mu \in \mathrm{M}\left(\mathscr{L}_{1}\right)$. If $\mathscr{L}_{2}$ coallocates $\mathscr{L}_{1}$, then $\exists \nu \in M_{R}\left(\mathscr{L}_{2}\right)$, s.t. $\mu \leq \nu$ on $\mathscr{L}_{1}$ and $\mu(X)=\nu(X)$.

\section{THEOREM 3.4 [Regular measure enlargement on a normal lattice]}

Suppose $\mathscr{L}$ is normal and $\mu \in \mathrm{M}(\mathscr{L})$. Then there exists a unique $\nu \in \mathrm{M}_{\mathrm{R}}(\mathscr{L})$, s.t. $\mu \leq \nu$ on $\mathscr{L}$ and $\mu(X)=\nu(X)$.

Furthermore, if we impose a $\sigma$-smoothness condition on $\mu$, we obtain the following :

\section{THEOREM 3.5}

Suppose $\mathscr{L}_{1} \subset \mathscr{L}_{2}$, and $\mathscr{L}_{2}$ coallocates $\mathscr{L}_{1}$, and $\mu \in M_{R}^{\sigma}\left(\mathscr{L}_{1}\right)$, 
$\nu \in M_{R}\left(\mathscr{L}_{2}\right)$, where $\nu$ is the regular measure extension of $\mu$. Then $\nu \in M_{R}\left(\mathscr{L}_{2}\right) \cap M_{\sigma}\left(\mathscr{L}_{2}^{\prime}\right)$.

Proof:

$$
\mu \in M_{R}^{\sigma}\left(\mathscr{L}_{1}\right) \Rightarrow \mu \in M_{R}\left(\varphi_{1}\right) \text { I hence by Theorem 3.2, }
$$

$\nu \equiv \mu^{\wedge} \mid \mathscr{L}_{2} \in M_{R}\left(\mathscr{L}_{2}\right)$ is the unique regular measure extension of $\mu$.

Theorem 3.1(7) $\Rightarrow \mu^{\wedge}$ is $\mathscr{L}_{1}$-regular on $\mathscr{L}_{2}^{\prime}$.

$\Rightarrow \forall \mathrm{B}_{\mathrm{n}}^{\prime} \in \mathscr{L}_{2}^{\prime}, \quad \mathrm{B}_{\mathrm{n}}^{\prime} \downarrow \emptyset, \quad \exists \mathrm{A}_{\mathrm{n}} \in \mathscr{L}_{1}, \quad \mathrm{~A}_{\mathrm{n}} \subset \mathrm{B}_{\mathrm{n}}^{\prime}, \quad \forall \varepsilon>0$,

$$
\mu^{\wedge}\left(B_{n}^{\prime}\right)<\mu^{\wedge}\left(A_{n}\right)+\varepsilon / 2
$$

$\mu^{\wedge}=\mu$ on $\mathscr{L}_{1}$, since $\mu$ is $\mathscr{L}_{1}$-regular [Theorem 3.1(5)[b]]

$$
\mu^{\wedge}\left(B_{n}^{\prime}\right)<\mu\left(A_{n}\right)+\varepsilon / 2
$$

since $A_{n} \subset B_{n}^{\prime} \downarrow \emptyset$, without the loss of generality, we may assume $A_{n} \downarrow \emptyset$. Thus, $\mu\left(A_{n}\right) \rightarrow 0 \quad \because \mu$ is $\sigma$-smooth on $\mathscr{L}_{1}$ Hence, $[\mathrm{i}] \Rightarrow \mu^{\wedge}\left(B_{n}^{\prime}\right)<\varepsilon$

$$
\text { Consequently, } \quad \mu^{\wedge}\left(\mathrm{B}_{\mathrm{n}}^{\prime}\right) \rightarrow 0 \quad \forall \mathrm{B}_{\mathrm{n}}^{\prime} \in \mathscr{L}_{2}^{\prime}
$$$$
\text { or, } \quad \nu=\mu^{\wedge} \mid \mathscr{L}_{2} \in M_{R}\left(\mathscr{L}_{2}\right) \cap M_{\sigma}\left(\mathscr{L}_{2}^{\prime}\right) \text {. }
$$

In particular, if $\mathscr{L}_{1}=\mathscr{L}_{2}=\mathscr{L}$ is normal, we have

\section{Corollary $\mathbf{3 . 5}$}

Suppose $\mathscr{L}$ is normal, and $\mu \in M_{\sigma}(\mathscr{L}), \nu \in M_{R}(\mathscr{L}), \nu$ is the regular measure enlargement of $\mu, \mu \leq \nu$ on $\mathscr{L}, \mu(X)=\nu(X)$. Then, $\nu \in M_{\sigma}\left(\mathscr{L}^{\prime}\right)$.

\section{THEOREM 3.6}

Suppose $\mathscr{L}_{1} \subset \mathscr{L}_{2}$, and $\mathscr{L}_{2}$ coallocates $\mathscr{L}_{1}$, and

$\mathscr{L}_{2}$ is countably paracompact and normal. Suppose $\mu \in \dot{M}_{R}^{\sigma}\left(\mathscr{L}_{1}\right)$, and $\nu \in M_{R}\left(\mathscr{L}_{2}\right)$, where $\nu$ is the unique regular measure extension of $\mu$. Then, $\quad \nu \in M_{R}^{\sigma}\left(\mathscr{L}_{2}\right)$.

Proof:

$$
\mathscr{L}_{2} \text { c.p. } \Rightarrow \forall \mathrm{B}_{\mathrm{n}} \in \mathscr{L}_{2}, \quad \mathrm{~B}_{\mathrm{n}} \downarrow \emptyset, \quad \exists \tilde{B}_{\mathrm{n}} \in \mathscr{L}_{2}, \quad \mathrm{~B}_{\mathrm{n}} \subset \tilde{B}_{\mathrm{n}}^{\prime} \downarrow \emptyset
$$

Theorem $3.5 \Rightarrow \nu \in M_{\sigma}\left(\mathscr{L}_{2}^{\prime}\right), \quad \nu\left(\tilde{B}_{n}^{\prime}\right) \rightarrow 0 \Rightarrow \nu\left(B_{n}\right) \rightarrow 0 \quad \forall B_{n} \in \mathscr{L}_{2}$ $\therefore \nu$ is $\sigma$-smooth on $\mathscr{L}_{2}$, and since $\nu$ is regular on $\mathscr{L}_{2}, \nu \in M_{R}^{\sigma}\left(\mathscr{L}_{2}\right)$.

We now give two applications of the results on coallocation lattices to topological spaces. 


\section{1) Measures on a locally compact Hausdroff space}

Let $X$ be a locally compact Hausdroff space and $\mathscr{L}_{1}=\mathrm{K}_{0}$ is the collection of all compact $\mathrm{G}_{\delta}$-sets, while $\mathscr{L}_{2}=\mathrm{K}$ is the collection of all compact sets. Note that in this case, $X$ does not belong to either $K_{0}$ or $K$, unless $X$ is compact. Then $K_{0} \subset K$, and it can be shown that $K$ coallocates $K_{0}$. For any $\mu \in M_{R}\left(K_{0}\right), \mu$ is $\sigma$-smooth, because $K_{0}$ is compact. Thus, $\mu \in M_{R}^{\sigma}\left(K_{0}\right)$. By the coallocation theorem, we can extend $\mu$ uniquely to a regular measure $\nu$ which is also $\sigma$-smooth, because $K$ is compact. Hence, $\nu \in M_{R}^{\sigma}(K)$.

\section{2) Measure enlargement from zero sets to closed sets}

suppose $X$ is a countably paracompact and normal topological space. Let $\mathscr{L}_{1}=3$ (zero sets) and $\mathscr{L}_{2}=\mathbf{s}$ (closed sets). That is, $s$ is c.p. normal. $\quad 3 \subset s$ because all zero sets are closed $G_{\delta}$-sets, and disjoint closed sets can be separated by disjoint zero sets. Therefore, 3 is c.p. and normal. Thus, 3 coseparates $\mathbf{s}$. Hence s coallocates 3 [Theorem 2.3].

Let $\mu \in M_{R}(3)$. Then by Theorem 3.2, there exists a unique regular measure extension $\nu \in M_{R}(\mathbf{s})$. Theorem 3.1(7)[a] implies $\nu$ on all open sets is 3 -regular.

Suppose $\mu \in M_{R}^{\sigma}(3)$. By Theorem 3.5, the unique regular measure extension is $\nu \in M_{R}(s) \cap M_{\sigma}\left(s^{\prime}\right)$. Now $s$ is c.p., hence $\nu \in M_{\sigma}(s)$ [Theorem 2.4]. Then, $\nu \in M_{R}^{\sigma}(s)$. This is the result of Marik [5].

\section{NORMAL LATTICES}

In this section, we give further characterization of normal lattices and further consequences of a lattice being normal in terms of associated measures on the generalized algebra.

\section{Definition 4.1}

Let $\mathscr{L}$ be a lattice of subsets of $X$, and $\mu \in M(\mathscr{L})$. $\forall E \subset X$, define

$$
\begin{aligned}
& \mu^{\prime}(E) \equiv \inf \left\{\mu\left(\tilde{L}^{\prime}\right): E \subset \tilde{L}^{\prime}, \tilde{L} \in \mathscr{L}\right\} \\
& \mu^{\prime \prime}(E) \equiv \inf \left\{\sum_{n=1}^{\infty} \mu\left(\tilde{L}_{n}^{\prime}\right): E \subset \bigcup_{n=1}^{\infty} \tilde{L}_{n}^{\prime}, \tilde{L}_{n} \in \mathscr{L}\right\} \\
& \mu^{\prime}(E) \equiv \sup \{\mu(\tilde{L}): E \supset \tilde{L} \in \mathscr{L}\} \\
& \mu^{\prime}(E) \equiv \inf \left\{\mu .\left(\tilde{L}^{\prime}\right): E \subset \tilde{L}^{\prime}, \tilde{L} \in \mathscr{L}\right\}
\end{aligned}
$$

It is clear that if $\mu \in M_{R}(\mathscr{L})$, then $\mu=\mu^{\prime}$ on $A(\mathscr{L})$. 


\section{TheOREM 4.1}

Proof:

Let $\mu, \nu \in M(\mathscr{L})$, such that $\mu(X)=\nu(X)$. Then

$\mu \leq \nu$ on $\mathscr{L} \Leftrightarrow \mu \leq \nu \leq \nu^{\prime} \leq \mu^{\prime}$ on $\mathscr{L}$

It is obvious that $\mu \leq \nu$ on $\mathscr{L} \Leftrightarrow \nu \leq \mu$ on $\mathscr{L}^{\prime}$. Let $\mathrm{E} \subset \mathrm{X}$ s.t.

$E \subset \tilde{L} \cdot, \tilde{L} \in \mathscr{L}, \nu\left(\tilde{L}^{\prime}\right) \leq \mu\left(\tilde{L}^{\prime}\right)$. Taking inf,

$\inf \left(\nu\left(\tilde{L}^{\prime}\right): \tilde{L}^{\prime} \in \mathscr{L}^{\prime}\right) \leq \inf \left(\mu\left(\tilde{L}^{\prime}\right): \tilde{L}^{\prime} \in \mathscr{L}^{\prime}\right)$,

$\therefore \nu^{\prime}(\mathrm{E}) \leq \mu^{\prime}(\mathrm{E})$. In particular, $\mathrm{E} \in \mathscr{L} \Rightarrow \nu^{\prime} \leq \mu^{\prime}$ on $\mathscr{L}$. Hence,

$\mu \leq \nu \leq \nu^{\prime} \leq \mu^{\prime}$ on $\mathscr{L}$.

\section{THEOREM 4.2}

$$
\begin{aligned}
& \text { Suppose } \forall \mu \in I(\mathscr{L}), \quad \mathrm{L}_{1}, \mathrm{~L}_{2} \in \mathscr{L}, \\
& \mu^{\prime}\left(\mathrm{L}_{1}\right)=1 \text { and } \mu^{\prime}\left(\mathrm{L}_{2}\right)=1 \quad \Rightarrow \quad \mu^{\prime}\left(\mathrm{L}_{1} \cap \mathrm{L}_{2}\right)=1
\end{aligned}
$$

Then, $\mathscr{L}$ is normal.

Proof:

Suppose $\mathscr{L}$ is not normal. Then

$\exists \mu \in I(\mathscr{L}), \quad \nu_{1}, \nu_{2} \in I_{\mathrm{R}}(\mathscr{L})$, s.t. $\mu \leq \nu_{1}$ on $\mathscr{L}, \mu \leq \nu_{2}$ on $\mathscr{L}$, but $\nu_{1} \neq \nu_{2}$.

$\therefore \exists \mathrm{L}_{1}, \mathrm{~L}_{2} \in \mathscr{L}, \quad \mathrm{L}_{1} \cap \mathrm{L}_{2}=\emptyset$,

$$
\nu_{1}\left(\mathrm{~L}_{1}\right)=1, \quad \nu_{2}\left(\mathrm{~L}_{1}\right)=0 \text { and } \nu_{1}\left(\mathrm{~L}_{2}\right)=0, \quad \nu_{2}\left(\mathrm{~L}_{2}\right)=1
$$

Now if $L_{1} \subset \tilde{L}_{1}^{\prime}, \tilde{L}_{1} \in \mathscr{L}$, then $\nu_{1}\left(\tilde{L}_{1}^{\prime}\right)=1$. Since

$\mu \leq \nu_{1}$ on $\mathscr{L} \Leftrightarrow \nu_{1} \leq \mu$ on $\mathscr{L}^{\prime}$, we have $\mu\left(\tilde{L}_{1}^{\prime}\right)=1 \Rightarrow \mu^{\prime}\left(\mathrm{L}_{1}\right)=1$.

similarly, if $\mathrm{L}_{2} \subset \tilde{L}_{2}^{\prime}, \tilde{\mathrm{L}}_{2} \in \mathscr{L}$, then $\mu^{\prime}\left(\mathrm{L}_{2}\right)=1$.

Then, by assumption, $\mu^{\prime}\left(L_{1} \cap L_{2}\right)=1$. But $L_{1} \cap L_{2}=\emptyset, \therefore \mu^{\prime}\left(L_{1} \cap L_{2}\right)=0$

gives a contradiction. Consequently, $\mathscr{L}$ is normal.

\section{THEOREM 4.3}

Let $\nu \in M_{R}(\mathscr{L}), \quad \rho \in M(\mathscr{L})$, s.t. $\nu(X)=\rho(X)$ and on $\mathscr{L}^{\prime}, \nu \leq \rho \in M_{R}\left(\mathscr{L}^{\prime}\right)$. Then

(1) $\rho \leq \nu=\nu^{\prime} \leq \rho$ on $\mathscr{L}$

(2) $\mathscr{L}$ is normal $\Rightarrow \nu=\nu^{\prime}=\rho^{\prime}$ on $\mathscr{L}$.

Proof:

(1) $\nu \leq \rho$ on $\mathscr{L}^{\prime} \Leftrightarrow \rho \leq \nu$ on $\mathscr{L}$, and $\nu \in M_{R}(\mathscr{L}) \Rightarrow \nu=\nu^{\prime}$. Hence

by Theorem 4.1, $\rho \leq \nu=\nu^{\prime} \leq \rho^{\prime}$ on $\mathscr{L}$.

(2) Suppose $\mathscr{L}$ is normal and $\exists \mathrm{L} \in \mathscr{L}$ s.t. $\nu(\mathrm{L})<\rho^{\prime}(\mathrm{L})$. $\nu \in \mathrm{M}_{\mathrm{R}}(\mathscr{L}) \Rightarrow \forall \varepsilon>0, \exists \tilde{\mathrm{L}} \in \mathscr{L}, \quad \tilde{\mathrm{L}} \subset \mathrm{L}^{\prime}, \nu(\tilde{\mathrm{L}})+\varepsilon>\nu\left(\mathrm{L}^{\prime}\right)$

$\therefore \nu\left(\tilde{L}^{\prime}\right)<\nu(L)+\varepsilon$ and $\operatorname{Ln} \tilde{L}=\emptyset$

By normality, $\exists \mathrm{L}_{\mathbf{a}}, \mathrm{L}_{\mathrm{b}} \in \mathscr{L}$, s.t. $\mathrm{L} \subset \mathrm{L}_{\mathrm{a}}^{\prime}, \tilde{\mathrm{L}} \subset \mathrm{L}_{\mathrm{b}}^{\prime}, \quad \mathrm{L}_{\mathrm{a}}^{\prime} \cap \mathrm{L}_{\mathrm{b}}^{\prime}=\emptyset$ 
$\therefore \quad \mathbf{L} \subset \mathbf{L}_{\mathbf{a}}^{\prime} \subset \mathbf{L}_{\mathbf{b}} \subset \tilde{\mathbf{L}}^{\prime}$

$\nu(L)<\rho^{\prime}(\mathrm{L}) \leq \rho^{\prime}\left(\mathrm{L}_{\mathrm{a}}^{\prime}\right) \underset{\mathscr{L}^{\prime}}{\underset{\rho}{=}} \rho\left(\mathrm{L}_{\mathrm{a}}^{\prime}\right) \leq \rho\left(\mathrm{L}_{\mathrm{b}}\right) \leq \underset{\mathscr{L}}{\leq} \nu\left(\mathrm{L}_{\mathrm{b}}\right) \leq \nu\left(\mathrm{L}^{\prime}\right)<\nu(\mathrm{L})+\varepsilon$

$\Rightarrow \rho^{\prime}(L) \leq \nu(L)$ gives a contradiction. $\therefore \nu=\nu^{\prime}=\rho^{\prime}$ on $\mathscr{L}$.

\section{THEOREM 4.4}

Let $\mathscr{L}$ be a lattice of subsets of $X$, and $\mu \in M_{\sigma}(\mathscr{L})$. Then,

(1) $\mu^{\prime \prime} \leq \mu^{\prime}$ everywhere

(2) $\mu^{\prime}=\mu$ on $\mathscr{L}^{\prime}$

(3) $\mu \leq \mu^{\prime \prime} \leq \mu^{\prime}$ on $\mathscr{L}$

(4) $\mu(X)=\mu^{\prime \prime}(X)=\mu^{\prime}(X)$

(5) $\mu_{.}\left(L^{\prime}\right)+\mu^{\prime}(L)=\mu(X), \quad \forall L \in \mathscr{L}$

(6) If $\mathscr{L}$ is normal, then $\mu \leq \mu^{\prime \prime} \leq \mu^{\prime}=\mu^{\prime}$ on $\mathscr{L}$

(7) If $\mathscr{L}$ is $\delta$-normal, then $\mu "=\mu$ on $\mathscr{L}$.

NOTE : The condition $\mu \in M_{\sigma}(\mathscr{L})$ is imposed, because when $\mu$ is a $0-1$ measure and if $\mu$ is not $\sigma$-smooth, then $\mu^{\prime \prime} \equiv 0$.

Proof:

(1) By definition of $\mu^{\prime \prime}$, the inf encompasses more sets than that of $\mu^{\prime}$, hence $\mu^{\prime \prime} \leq \mu^{\prime}$ everywhere.

(2) Take $\mathrm{E}=\tilde{L}^{\prime} \in \mathscr{L}^{\prime}, \therefore \mu^{\prime}=\mu$ on $\mathscr{L}^{\prime}$. In particular,

$$
\mu^{\prime}(X)=\mu(X)
$$

(3) From (1) and [i], we have $\mu^{\prime \prime}(X) \leq \mu(X)$. We now show that $\mu^{\prime \prime}(X)=\mu(X)$. For suppose $X=\bigcup_{i=1}^{\infty} L_{i}^{\prime}$, pairwise disjoint $L_{i}^{\prime} \in \mathscr{L}{ }^{\prime}$, and $\quad \sum_{i=1}^{\infty} \mu\left(L_{i}^{\prime}\right)<\mu(X)$, but $\sum_{i=1}^{\infty} \mu\left(L_{i}^{\prime}\right)=\lim _{n \rightarrow \infty} \sum_{i=1}^{n} \mu\left(L_{i}^{\prime}\right)$ $\geq \lim _{n \rightarrow \infty} \mu\left(\bigcup_{i=1}^{n} L_{i}^{\prime}\right)=\mu(X)$.

Since $U_{i=1}^{n} L_{i}^{\prime} \in \mathscr{L}$ and $\bigcup_{i=1}^{n} L_{i}{ }^{\prime} \uparrow$, or $\prod_{i=1}^{\infty} L_{i} \downarrow \emptyset$, also $\mu \in M_{\sigma}(\mathscr{L})$. Taking the inf of the above, we have $\mu^{\prime \prime}(X) \geq \mu(X)$. Consequently, $\quad \mu "(X)=\mu(X)$ [ii] Now suppose $\exists L \in \mathscr{L}, \mu(L)>\mu "(L)$,

$$
\begin{aligned}
\mu^{\prime \prime}(\mathrm{X})=\mu^{\prime \prime}\left(\mathrm{L} \cup \mathrm{L}^{\prime}\right) & \leq \mu^{\prime \prime}(\mathrm{L})+\mu^{\prime \prime}\left(\mathrm{L}^{\prime}\right) & & \\
& \leq \mu^{\prime \prime}(\mathrm{L})+\mu\left(\mathrm{L}^{\prime}\right) & & \left(\because \mu^{\prime \prime} \leq \mu \text { on } \mathscr{L}^{\prime}\right) \\
& <\mu(\mathrm{L})+\mu\left(\mathrm{L}^{\prime}\right) & & (\text { by assumption }) \\
& =\mu(\mathrm{X}) & & \text { contradicting [ii] }
\end{aligned}
$$

$\therefore \mu \leq \mu$ " on $\mathscr{L}$. Together with (1), we have $\mu \leq \mu " \leq \mu$ on $\mathscr{L}$.

(4) [i] and [ii] $\Rightarrow \mu(X)=\mu^{\prime \prime}(X)=\mu^{\prime}(X)$. 
(5) $\forall \varepsilon>0, \quad \mu .\left(L^{\prime}\right)-\varepsilon<\mu(\hat{L})$ where $L^{\prime} \supset \hat{\mathrm{L}} \in \mathscr{L}$ $\therefore \quad \mu(X)-\mu_{0}\left(L^{\prime}\right)>\mu\left(\hat{L}^{\prime}\right)-\varepsilon$

Taking inf, we have

$$
\mu(X)-\mu_{.}\left(L^{\prime}\right) \geq \mu^{\prime}(L)
$$

If $L^{\prime} \supset \hat{L}, \mu .\left(L^{\prime}\right) \geq \mu\left(L^{\prime}\right) \geq \mu(\hat{L})$ since $\mu . \leq \mu$ on $\mathscr{L}^{\prime}$

$\therefore \mu(X)-\mu_{0}\left(L^{\prime}\right) \leq \mu(X)-\mu(\hat{L})=\mu(\hat{L} \cdot)$

Taking inf, we have

$$
\mu(X)-\mu_{.}\left(L^{\prime}\right) \leq \inf \left(\mu\left(\hat{L}^{\prime}\right): L \subset \hat{L}^{\prime}, \hat{\mathbf{L}} \in \mathscr{L}\right)=\mu^{\prime}(\mathrm{L}) \quad \ldots . . \text { [iv] }
$$

[iii] and [iv] $\Rightarrow \mu(X)-\mu_{0}\left(L^{\prime}\right)=\mu^{\prime}(L) \quad \forall L \in \mathscr{L}$.

(6) $\mathrm{L}$ is normal, then Corollary 3.1(2) and (5) imply $\mu^{\wedge}(L)=\mu^{\prime}(L) \quad \forall L \in \mathscr{L}$. Now $\mu^{\prime}=\mu^{\wedge}$ on $\mathscr{L}$, and from (3), $\mu \leq \mu^{\prime \prime} \leq \mu^{\prime}=\mu^{\wedge}$ on $\mathscr{L}$.

(7) From (1), $\mu^{\prime \prime} \leq \mu^{\prime}$ everywhere. Suppose $\exists L \in \mathscr{L}$, s.t. $\mu^{\prime \prime}(L)<\mu^{\prime}(L)$

Then $L \subset \bigcup_{n=1}^{\infty} L_{n}^{\prime}, L_{n} \in \mathscr{L} . \quad \mathscr{L}$ is $\delta \Rightarrow \prod_{n=1}^{\infty} L_{n} \in \mathscr{L}$.

Let $D=\bigcap_{n=1}^{\infty} L_{n}, L \cap D=\emptyset . \mathscr{L}$ is normal $\Rightarrow \exists A, B \in \mathscr{L}$, s.t.

$L \subset A^{\prime}, D^{\prime} \subset B^{\prime}, A^{\prime} \cap B^{\prime}=\emptyset \quad \therefore \quad L \subset A^{\prime} \subset B^{\prime} \subset D^{\prime}=\bigcup_{n=1}^{\infty} L_{n}^{\prime}$

$\mu^{\prime}(L) \leq \mu^{\prime}\left(A^{\prime}\right) \underset{\text { from (2) }}{=} \mu\left(A^{\prime}\right) \leq \mu(B) \underset{\text { from (3) }}{\leq} \mu^{\prime \prime}(B) \leq \sum_{n=1}^{\infty} \mu\left(L_{n}^{\prime}\right)$

Taking inf, and since $L \subset \bigcup_{n=1}^{\infty} L_{n}^{\prime}$,

$\mu^{\prime}(L) \leq \inf \left\{\sum_{n=1}^{\infty} \mu\left(L_{n}^{\prime}\right): L \subset \sum_{n=1}^{\infty} L_{n}^{\prime}, L_{n} \in \mathscr{L}\right\} \equiv \mu^{\prime \prime}(L)$

gives a contradiction. $\therefore \mu^{\prime \prime}(L)=\mu^{\prime}(L)$, or $\mu^{\prime \prime}=\mu^{\prime}$ on $\mathscr{L}$.

\section{Theorem 4.5}

Let $\mathscr{L}$ be a lattice of subsets of $X$, and let $\mu \in M_{\sigma}(\mathscr{L}), \rho \in M(\mathscr{L})$, s.t. $\mu \leq \rho$ on $\mathscr{L}, \mu(X)=\rho(X)$.

If $\mathscr{L}$ is countably paracompact and normal, then $\rho \in M_{\sigma}(\mathscr{L})$.

Proof:

Let $\mathrm{L}_{\mathrm{n}} \downarrow \emptyset, \quad \mathrm{L}_{\mathrm{n}} \in \mathscr{L}, \quad \forall \mathrm{n}$

$\mathscr{L}$ c.p. $\Rightarrow \exists \tilde{\mathrm{L}}_{\mathrm{n}} \in \mathscr{L}, \quad \mathrm{L}_{\mathrm{n}} \subset \tilde{\mathrm{L}}_{\mathrm{n}}^{\prime} \downarrow \emptyset \quad \therefore \mathrm{L}_{\mathrm{n}} \cap \tilde{\mathrm{L}}_{\mathrm{n}}=\emptyset$

$\mathscr{L}$ normal $\Rightarrow \exists A_{n}, B_{n} \in \mathscr{L}, \quad L_{n} \subset A_{n}^{\prime}, \quad i_{n} \subset B_{n}^{\prime}, A_{n}^{\prime} \cap B_{n}^{\prime}=\emptyset$.

or, $\quad L_{n} \subset A_{n}^{\prime} \subset B_{n} \subset L_{n}^{\prime} \downarrow \emptyset, \quad \therefore \rho\left(L_{n}\right) \leq \rho\left(A_{n}^{\prime}\right) \leq \mu\left(A_{n}^{\prime}\right) \leq \mu\left(B_{n}\right) \rightarrow 0$

(one may assume, with the loss of generality, $B_{n} \downarrow$ ).

$\left(\because \rho \leq \mu\right.$ on $\mathscr{L}^{\prime} ; \mathrm{B}_{\mathrm{n}} \downarrow \emptyset$ and $\left.\mu \in M_{\sigma}(\mathscr{L})\right) \quad \therefore \rho\left(L_{n}\right) \rightarrow 0$, or $\rho \in M_{\sigma}(\mathscr{L})$.

\section{Theorem $\mathbf{4 . 6}$}

Proof:

Suppose $\mathscr{L}_{1} \subset \mathscr{L}_{2}$, and $\mathscr{L}_{1}$ separates $\mathscr{L}_{2}$. Then, $\mathscr{L}_{1}$ normal $\Leftrightarrow \mathscr{L}_{2}$ normal.

$" \Rightarrow "$ Suppose $\mathscr{L}_{1}$ is normal. 
Let $\mu \in \mathrm{I}\left(\mathscr{L}_{2}\right), \quad \nu_{\mathrm{a}}, \nu_{\mathrm{b}} \in \mathrm{I}_{\mathrm{R}}\left(\mathscr{L}_{2}\right)$, s.t. $\mu \leq \nu_{\mathrm{a}}$ on $\mathscr{L}_{2}, \mu \leq \nu_{\mathrm{b}}$ on $\mathscr{L}_{2}$. Then $\mu\left|\in I\left(\mathscr{L}_{1}\right), \nu_{\mathrm{a}}\right|, \nu_{\mathrm{b}} \mid \in \mathrm{I}_{\mathrm{R}}\left(\mathscr{L}_{1}\right)$, and $\mu\left|\leq \nu_{\mathrm{a}}\right|$ on $\mathscr{L}_{1}, \mu\left|\leq \nu_{\mathrm{b}}\right|$ on $\mathscr{L}_{1} \cdot \mathscr{L}_{1}$ normal $\Leftrightarrow \nu_{\mathrm{a}}\left|=\nu_{\mathrm{b}}\right|$ [Theorem 2.2].

Extend $\nu_{\mathrm{a}} \mid$ and $\nu_{\mathrm{b}} \mid$ to $\mathscr{L}_{2} \Rightarrow \nu_{\mathrm{a}}=\nu_{\mathrm{b}} \Leftrightarrow \mathscr{L}_{2}$ normal,

$\because \mathscr{L}_{1}$ separates $\mathscr{L}_{2}$, the extension is unique.

"६" Suppose $\mathscr{L}_{2}$ is normal.

Let $\mu \in I\left(\mathscr{L}_{1}\right), \quad \nu_{\mathrm{a}}, \nu_{\mathrm{b}} \in \mathrm{I}_{\mathrm{R}}\left(\mathscr{L}_{1}\right)$, s.t. $\mu \leq \nu_{\mathrm{a}}$ on $\mathscr{L}_{1}, \mu \leq \nu_{\mathrm{b}}$ on $\mathscr{L}_{1}$.

Extend $\mu$ to $\lambda \in I\left(\mathscr{L}_{2}\right)$, and $\nu_{a}, \nu_{b}$ to $r_{a}, r_{b} \in I_{R}\left(\mathscr{L}_{2}\right)$,

respectively. We now show that $\lambda \leq \tau_{2}$ on $\mathscr{L}_{2}$, and $\lambda \leq \tau_{b}$ on $\mathscr{L}_{2}$.

For suppose $\exists \mathrm{L}_{2} \in \mathscr{L}_{2}$ s.t. $\lambda\left(\mathrm{L}_{2}\right)=1$ but $\tau_{\mathrm{a}}\left(\mathrm{L}_{2}\right)=0$. Then

$\tau_{2}\left(L_{2}^{\prime}\right)=1$. But $r_{\mathrm{a}} \in \mathrm{I}_{\mathrm{R}}\left(\mathscr{L}_{2}\right), \exists \tilde{\mathrm{L}}_{2} \in \mathscr{L}_{2}$, s.t. $\tilde{\mathrm{L}}_{2} \subset \mathrm{L}_{2}^{\prime}, \quad \tau_{2}\left(\tilde{\mathrm{L}}_{2}\right)=1$

since $\mathscr{L}_{1}$ separates $\mathscr{L}_{2} \Rightarrow \exists \mathrm{L}_{1} \in \mathscr{L}_{1}$, s.t. $\mathrm{L}_{2} \subset \mathrm{L}_{1} \subset \tilde{L}_{2}^{\prime}$

$\therefore 1=\lambda\left(L_{2}\right) \leq \lambda\left(L_{1}\right) \underset{\mathscr{L}_{1}}{=} \mu\left(L_{1}\right) \underset{\mathscr{L}_{1}}{\leq} \nu_{2}\left(L_{1}\right) \underset{\mathscr{L}_{1}}{=} \tau_{2}\left(L_{1}\right) \leq \tau_{2}\left(\tilde{L}_{2}^{\prime}\right)$

Thus, $r_{\mathrm{a}}\left(\tilde{L}_{2}^{\prime}\right)=1$ or $r_{2}\left(\tilde{L}_{2}\right)=0$ contradicting $r_{2}\left(\tilde{L}_{2}\right)=1$

$\therefore \lambda \leq \tau_{\mathrm{a}}$ on $\mathscr{L}_{2}$. Similarly, $\lambda \leq \tau_{\mathrm{b}}$ on $\mathscr{L}_{2}$. Since $\mathscr{L}_{2}$ is normal,

$\tau_{\mathrm{a}}=\tau_{\mathrm{b}} \quad \therefore \tau_{\mathrm{a}}\left|=\tau_{\mathrm{b}}\right|$, i.e. $\nu_{\mathrm{a}}=\nu_{\mathrm{b}} \Leftrightarrow \mathscr{L}_{1}$ is normal.

\section{THEOREM 4.7}

Suppose $\mathscr{L}_{1} \subset \mathscr{L}_{2}$, and $\mu \in M_{R}\left(\mathscr{L}_{1}\right), \quad \nu \in M_{R}\left(\mathscr{L}_{2}\right)$, s.t. $\mu(X)=\nu(X), \nu \mid \mathscr{L}_{1}=\mu$. Then

Proof: $\quad \mathscr{L}_{1}$ separates $\mathscr{L}_{2} \Rightarrow \nu$ is $\mathscr{L}_{1}$-regular on $\mathscr{L}_{2}^{\prime}$. $\nu \in \mathrm{M}_{\mathrm{R}}\left(\mathscr{L}_{2}\right), \therefore \forall \mathrm{L}_{2}^{\prime} \in \mathscr{L}_{2}^{\prime}, \quad \nu\left(\mathrm{L}_{2}^{\prime}\right)=\sup \left\{\nu\left(\tilde{\mathrm{L}}_{2}\right): \mathrm{L}_{2}^{\prime} \supset \tilde{\mathrm{L}}_{2} \in \mathscr{L}_{2}\right\}$

$\forall \varepsilon>0, \quad \mathrm{~L}_{2}^{\prime} \supset \tilde{\mathrm{L}}_{2} \in \mathscr{L}_{2}, \quad \nu\left(\mathrm{L}_{2}^{\prime}\right)<\nu\left(\tilde{\mathrm{L}}_{2}\right)+\varepsilon$

$\mathrm{L}_{2} \cap \tilde{\mathrm{L}}_{2}=\emptyset$, and $\mathscr{L}_{1}$ separates $\mathscr{L}_{2} \Rightarrow$

$\exists \mathrm{L}_{1}, \tilde{\mathrm{L}}_{1} \in \mathscr{L}_{1}$, s.t. $\mathrm{L}_{2} \subset \mathrm{L}_{1}, \tilde{\mathrm{L}}_{2} \subset \tilde{\mathrm{L}}_{1}, \mathrm{~L}_{1} \cap \tilde{\mathrm{L}}_{1}=\emptyset$

$$
\begin{aligned}
\nu\left(\mathrm{L}_{2}^{\prime}\right) & <\nu\left(\tilde{\mathrm{L}}_{2}\right)+\varepsilon \\
& \leq \nu\left(\tilde{\mathrm{L}}_{1}\right)+\varepsilon \quad\left(\tilde{\mathrm{L}}_{2} \subset \tilde{\mathrm{L}}_{1}\right) \\
& =\mu\left(\tilde{\mathrm{L}}_{1}\right)+\varepsilon \quad\left(\nu \mid \mathscr{L}_{1}=\mu\right)
\end{aligned}
$$

Taking sup, $\nu\left(L_{2}^{\prime}\right)=\sup \left(\mu\left(\tilde{L}_{1}\right): L_{2}^{\prime} \supset \tilde{L}_{1} \in \mathscr{L}_{1}\right), \forall L_{2}^{\prime} \in \mathscr{L}_{2}^{\prime}$

i.e. $\nu$ is $\mathscr{L}_{1}$-regular on $\mathscr{L}_{2}^{\prime}$. 


\section{ACKNOWLEDGEMENT}

I express my gratitude to my teacher and dissertation advisor, Professor George Bachman of Polytechnic University (Brooklyn, NY), for his guidance, encouragement, and proof reading of this paper. I would also like to thank the two Referees for their valuable comments.

\section{REFERENCES}

[1] A.D. Alexandroff, Additive set functions in abstract spaces, Mat. Sb. (N.S.), 9(51), (1941), 563-628.

[2] J. Camacho, Jr., Extensions of lattice regular measures with applications, J. Indian Math. Soc., 54, (1989), 233-244.

[3] G. Eid, On normal lattices and Wallman spaces, Internat. J. Math. \& Math. Sci., 13, No.1, (1990), 31-38.

[4] P. Grassi, On subspaces of replete and measure replete spaces, Canad. Math. Bull., 27, (1984), 58-64.

[5] J. Marik, The Baire and Borel measures, Czech. J. Math., 7, (1957), 248-253.

[6] G. Nöbeling, Grundlagen der Analytischen Topologie, Springer-Verlag, Berlin, 1954.

[7] M. Szeto, Measure repleteness and mapping preservations, J. Indian Math. Soc., 43, (1979), $35-52$.

[8] M. Szeto, On maximal measures with respect to a lattice, Measure Theory and Applications, Proceedings of the 1980 Conference, Northern Illinois University, (1981), 277-282.

[9] H. Wallman, Lattices and topological spaces, Ann. of Math., 39, No.1, (1938), 112-126. 


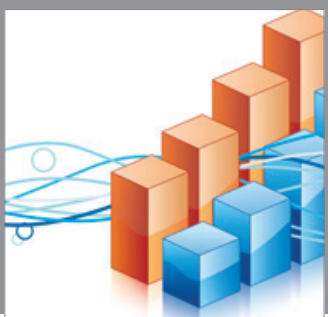

Advances in

Operations Research

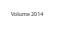

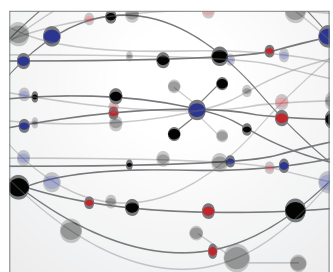

\section{The Scientific} World Journal
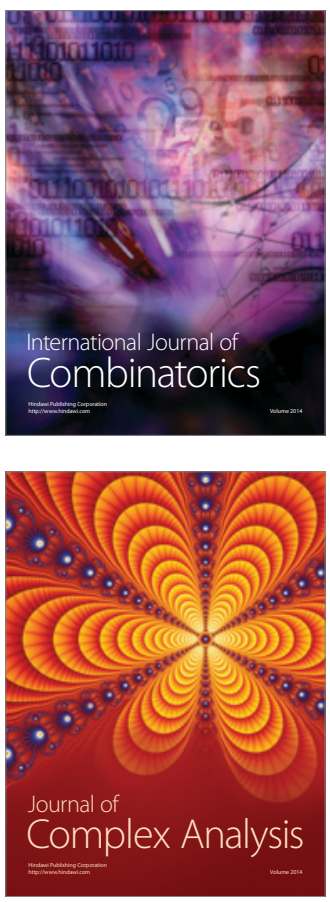

International Journal of

Mathematics and

Mathematical

Sciences
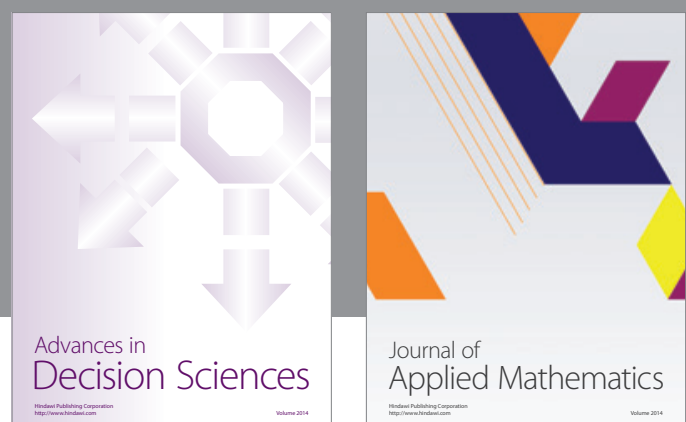

Journal of

Applied Mathematics
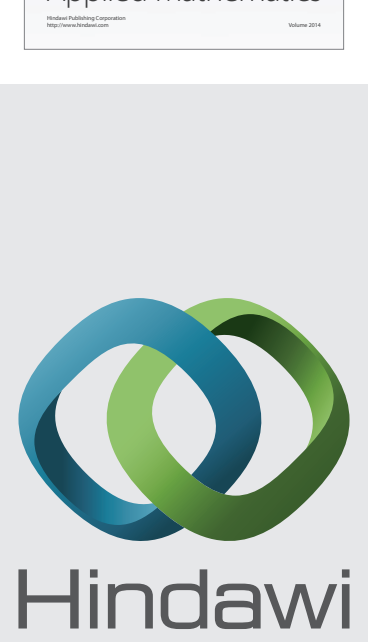

Submit your manuscripts at http://www.hindawi.com
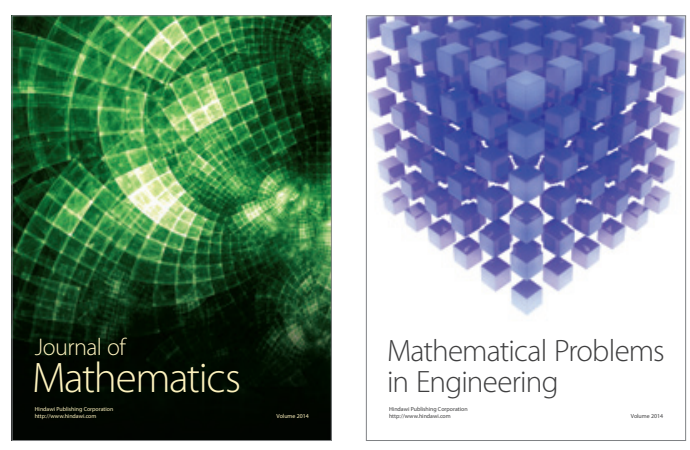

Mathematical Problems in Engineering
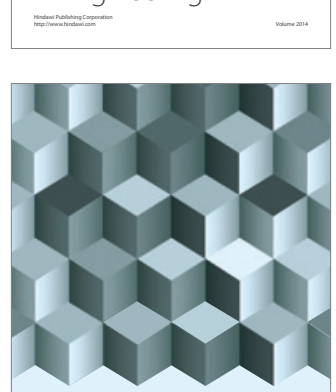

Journal of

Function Spaces
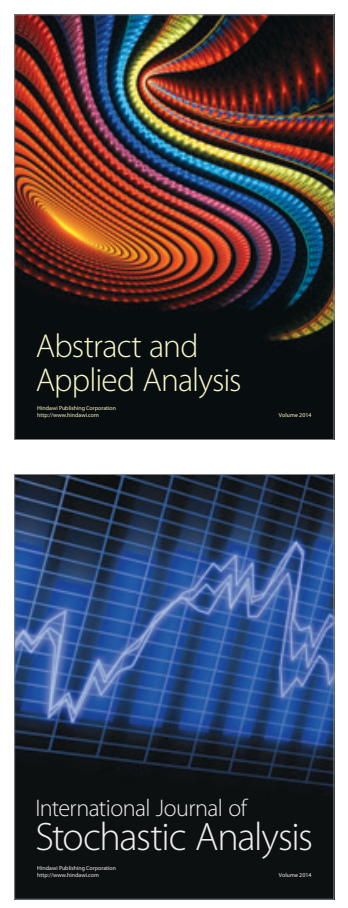

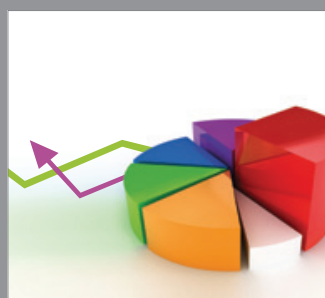

ournal of

Probability and Statistics

Promensencen
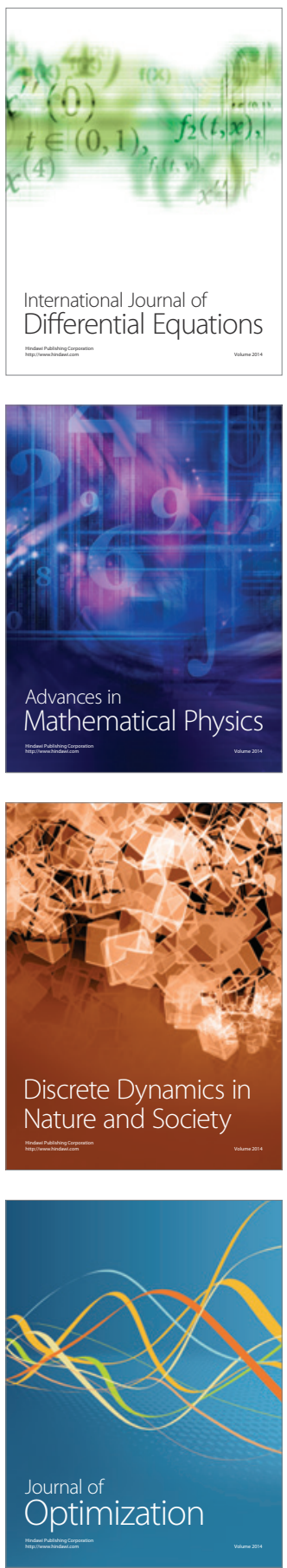